TRANSACTIONS OF THE

AMERICAN MATHEMATICAL SOCIETY

Volume 358, Number 11, November 2006, Pages 4843-4872

S 0002-9947(06)04189-4

Article electronically published on June 9, 2006

\title{
$W^{2, p}$-ESTIMATES FOR THE LINEARIZED MONGE-AMPÈRE EQUATION
}

\author{
CRISTIAN E. GUTIÉRREZ AND FEDERICO TOURNIER
}

\begin{abstract}
Let $\Omega \subseteq \mathbb{R}^{n}$ be a strictly convex domain and let $\phi \in C^{2}(\Omega)$ be a convex function such that $\lambda \leq \operatorname{det} D^{2} \phi \leq \Lambda$ in $\Omega$. The linearized MongeAmpère equation is

$$
L_{\Phi} u=\operatorname{trace}\left(\Phi D^{2} u\right)=f
$$

where $\Phi=\left(\operatorname{det} D^{2} \phi\right)\left(D^{2} \phi\right)^{-1}$ is the matrix of cofactors of $D^{2} \phi$. We prove that there exist $p>0$ and $C>0$ depending only on $n, \lambda, \Lambda$, and $\operatorname{dist}\left(\Omega^{\prime}, \Omega\right)$ such that

$$
\left\|D^{2} u\right\|_{L^{p}\left(\Omega^{\prime}\right)} \leq C\left(\|u\|_{L^{\infty}(\Omega)}+\|f\|_{L^{n}(\Omega)}\right)
$$

for all solutions $u \in C^{2}(\Omega)$ to the equation $L_{\Phi} u=f$.
\end{abstract}

\section{INTRODUCTION}

The $L^{p}$-estimates for the second derivatives of solutions to second order elliptic nondivergence equations of the form trace $\left(A(x) D^{2} u(x)\right)=f(x)$ (where the matrix $A(x)$ is uniformly elliptic in a domain $\Omega \subset \mathbb{R}^{n}$, that is, $\lambda|\xi|^{2} \leq\langle A(x) \xi, \xi\rangle \leq \Lambda|\xi|^{2}$ for all $x \in \Omega$ and $\xi \in \mathbb{R}^{n}$ ) were derived in the 1950's as a consequence of the CalderónZygmund theory of singular integrals. More precisely, if $A(x)$ is continuous in $\Omega$, then for any domain $\Omega^{\prime} \Subset \Omega$ and any $1<p<\infty$ we have

$$
\left\|D^{2} u\right\|_{L^{p}\left(\Omega^{\prime}\right)} \leq C\left(\|u\|_{L^{p}(\Omega)}+\|f\|_{L^{p}(\Omega)}\right),
$$

where $C$ is a constant depending only on $n, p, \lambda, \Lambda$, $\operatorname{dist}\left(\Omega^{\prime}, \partial \Omega\right)$ and the modulus of continuity of the matrix $A(x)$; see [GT83, Chapter 9].

If the coefficient matrix $A$ is uniformly elliptic and only measurable, then it was discovered by Lin [L86] that the inequality

$$
\left\|D^{2} u\right\|_{L^{p}\left(\Omega^{\prime}\right)} \leq C\left(\|u\|_{L^{\infty}(\Omega)}+\|f\|_{L^{n}(\Omega)}\right)
$$

holds true for some $p>0$, possibly small, and $C>0$ depends only on $n, \lambda, \Lambda$, $\operatorname{dist}\left(\Omega^{\prime}, \partial \Omega\right)$, and it is false for any $p$. An estimate similar to (1.2) was discovered by Evans [E85] for fully nonlinear uniformly elliptic equations of the form $F\left(D^{2} u\right)=0$.

Recently, Caffarelli proved that if $u$ is convex in $\Omega$ convex normalized, $f$ is continuous with $0<\lambda \leq f \leq \Lambda$ in $\Omega$, and $u$ solves the Monge-Ampère equation $\operatorname{det} D^{2} u=f$ with $u=0$ on $\partial \Omega$, then $\left\|D^{2} u\right\|_{L^{p}\left(\Omega^{\prime}\right)} \leq C$ for all $p>0$ with a constant $C$ depending only on $n, p, \lambda, \Lambda$, $\operatorname{dist}\left(\Omega^{\prime}, \partial \Omega\right)$, and the modulus of continuity of $f$; see C] and [G01, Chapter 6].

Received by the editors August 19, 2004.

2000 Mathematics Subject Classification. Primary 35B45, 35J60, 35J70.

Key words and phrases. A priori estimates of second derivatives, cross sections of solutions, viscosity solutions, nonuniformly elliptic equations.

The first author was partially supported by NSF grant DMS-0300004.

(C)2006 American Mathematical Society 4843

Reverts to public domain 28 years from publication 
In this paper we consider the linearized Monge-Ampère operator. We stress that this operator is in general not uniformly elliptic in $\Omega$. More precisely, let $\Omega \subseteq \mathbb{R}^{n}$ be a strictly convex domain and let $\phi \in C^{2}(\Omega)$ be a convex function such that $\lambda \leq \operatorname{det} D^{2} \phi \leq \Lambda$ in $\Omega$. The linearized Monge-Ampère equation is

$$
L_{\Phi} u=\operatorname{trace}\left(\Phi D^{2} u\right)=f
$$

where $\Phi=\left(\operatorname{det} D^{2} \phi\right)\left(D^{2} \phi\right)^{-1}$ is the matrix of cofactors of $D^{2} \phi$. A theory for this equation has been developed in [CG97] where, in particular, Harnack's inequality is proved. Applications of this theory include the solution of a problem in affine differential geometry; see [TW00].

The purpose of this paper is to study the $L^{p}$ integrability of second derivatives of solutions to (1.3). The main result of the paper, Theorem 6.3, is that there exist $p>0$ and $C>0$ depending only on $n, \lambda, \Lambda$, and $\operatorname{dist}\left(\Omega^{\prime}, \Omega\right)$ such that

$$
\left\|D^{2} u\right\|_{L^{p}\left(\Omega^{\prime}\right)} \leq C\left(\|u\|_{L^{\infty}(\Omega)}+\|f\|_{L^{n}(\Omega)}\right)
$$

for all solutions $u \in C^{2}(\Omega)$ to (1.3). In addition, the estimate (1.4) is false for all $p>0$. Indeed, we show in Section 8 that given $p>1$, there exist $\lambda(p)$ and $\Lambda(p)$ and a sequence $\phi_{n} \in C^{\infty}(\Omega)$ such that $\phi=0$ on $\partial \Omega$ and $\lambda \leq \operatorname{det} D^{2} \phi_{n} \leq \Lambda$ for each $n$ and such that $\left\|D^{2} \phi_{n}\right\|_{L^{p}\left(B_{\delta}(0)\right)} \rightarrow \infty$ as $n \rightarrow \infty$.

One important element in the analysis of $(1.3)$ is the fact that $L_{\Phi} \phi \approx 1$, which means that the function $\phi$ takes the role that quadratic polynomials take on a uniformly elliptic equation. The analogous sets to Euclidean balls in this setting are the level sets of the function $\phi$ which are called sections. The main idea to prove our result is to separate the problem into two main steps. The first step is to estimate the measure of the set of points where a solution $u$ can be approximated by quadratic polynomials in which the quadratic term is defined by the quasidistance given by the sections of $\phi$ (see Definition 3.2); a covering theorem with sections is crucial in this step. The second step consists of estimating the measure of the set of points where the quasidistance is dominated by Euclidean distance; this step deals only with the convex function $\phi$.

To prove our theorem we introduce the sets $G_{M}(u)$ given in Definition 3.5 in terms of the quasidistance $d$ which takes into account the behavior of $\phi$ in different directions. Once we define these sets, a difficulty comes from deciding the size of the neighborhood in which we want the inequality in the definition of $G_{M}(u)$ to hold. To estimate $D^{2} u\left(x_{0}\right)$ it is enough to have

$$
\left|u(x)-u\left(x_{0}\right)-\nabla(u)\left(x_{0}\right)\left(x-x_{0}\right)\right| \leq M d\left(x, x_{0}\right)^{2}
$$

for all $x$ in a neighborhood of $x_{0}$, but it is important to specify this neighborhood since eventually we have to compare $G_{M}(u)$ with $G_{\lambda M}(u)$; in particular this becomes important in the hypothesis of Lemma 4.2

Our proof uses the method developed by Caffarelli [C] to establish the $W^{2, p_{-}}$ estimates for the Monge-Ampère equation; see also [G01, Chapter 6]. Some of these ideas are also used in the $W^{2, p}$-estimates for nonlinear elliptic equations in Caffarelli and Cabré's book [CC95] from where some of the notation is borrowed.

The method of proof differs considerably from the classical $W^{2, p}$-estimates as in Gilbarg and Trudinger GT83 as well as the estimates of Lin and Evans. Gilbarg and Trudinger's proof uses perturbation arguments and is based on the inequality $\left\|D^{2} u\right\|_{L^{p}(\Omega)} \leq C\|\Delta u\|_{L^{p}(\Omega)}$ for $u \in W_{0}^{2, p}(\Omega)$. The ellipticity of the operator $L$ and 
the modulus of continuity of the coefficients of $L$ are essential in that proof; these two elements are not present in our setting.

A hypothesis requires that $\lambda \leq \operatorname{det} D^{2} \phi \leq \Lambda$, and our method does not seem to give information about the relation between the best exponent $p$, for which our theorem holds the constants $\lambda$ and $\Lambda$. A conjecture is that if $\left|\operatorname{det} D^{2} \phi-1\right| \leq \epsilon$, then the integrability exponent $p(\epsilon)$ in (1.4) tends to $\infty$ as $\epsilon \rightarrow 0$. It is true that if $\left|\operatorname{det} D^{2} \phi-1\right| \leq \epsilon$ with $\phi=0$ on $\partial \Omega$ normalized, then $\left\|D^{2} \phi\right\|_{L^{p}\left(\Omega_{1 / 2}\right)} \leq C$, where $p(\epsilon)$ tends to $\infty$ as $\epsilon$ tends to 0 . In some sense this says that the "ellipticity becomes better" when $\operatorname{det} D^{2} \phi$ is closer to one, but it does not seem to give information on the modulus of continuity of the coefficients of our equation.

The organization of this paper is the following. In Section 2 we introduce all the preliminary material. Section 3 contains the main definitions, and the second step mentioned above is proved. Section 4 contains the proof of the density lemmas needed later. In Section 5 we prove the main result for the homogeneous case, and Section 6 contains the nonhomogeneous case. Section 7 contains the proof of an inequality used in Section 3 , and Section 8 contains the examples mentioned in this Introduction.

\section{Preliminary Results}

In this section we list the results about sections and normalization that are relevant for what follows.

Given a function $\phi: \Omega \rightarrow \mathbb{R}, \partial \phi$ denotes the subdifferential of $\phi$. The MongeAmpère measure associated with $\phi$ is

$$
\mu(E)=M \phi(E)=|\partial \phi(E)|
$$

for all Borel subsets $E \subset \Omega$. In case $\phi$ is convex and $C^{2}(\Omega)$, we have

$$
|\partial \phi(E)|=\int_{E} \operatorname{det} D^{2} \phi(y) d y .
$$

A convex normalized domain is a strictly convex domain $\Omega \subseteq \mathbb{R}^{n}$ such that $B_{\alpha_{n}}(0) \subseteq$ $\Omega \subseteq B_{1}(0)$. If $S$ is any convex set with nonempty interior, there exists an ellipsoid $E$ such that $\alpha_{n} E \subseteq S \subseteq E$, and hence there exists an affine transformation $T$ such that $B_{\alpha_{n}}(0) \subseteq T(S) \subseteq B_{1}(0)$.

A section of the convex function $\phi \in C^{1}(\Omega)$ is defined by

$$
S_{\phi}(\bar{x}, t)=\{x \in \Omega: \phi(x)<\phi(\bar{x})+\nabla \phi(\bar{x}) \cdot(x-\bar{x})+t\},
$$

where $\nabla$ denotes the standard gradient.

Given $0<\alpha<1$ we define the sections at the minimum to be the sets

$$
\Omega_{\alpha}=\left\{x \in \Omega: \phi(x)<(1-\alpha) \min _{\Omega} \phi\right\} .
$$

The next five results about sections hold under the assumption that $\phi \in C^{2}(\Omega)$ is a convex function such that $\lambda \leq \operatorname{det}\left(D^{2} \phi\right) \leq \Lambda$ and $\phi=0$ on $\partial \Omega$, where $\Omega$ is a convex normalized domain. These results can be found in [G01, Chapters 3 and 5].

Lemma 2.1. For each $0<\alpha<1$ there exists $\eta$ depending only on $\alpha, \lambda$ and $\Lambda$, such that for all $x \in \Omega_{\alpha}$ and for all $t \leq \eta$, we have $S_{\phi}(x, t) \Subset \Omega$.

Lemma2.1 is crucial in two ways for our result. First, because as we mentioned in the Introduction, it allows for sections to take the role of Euclidean balls, and certain very important sets are defined using sections. Second, because the exponent $p$ appearing in our main theorem depends on the constant $\eta$ of this lemma. 
It is also important to remark that Lemma 2.1 holds under weaker assumptions on the function $\phi$, but certain regularity of $\phi$ is required on $\partial \Omega$ as examples of Pogorelov show; see [G01, Chapter 5]. We will only use this lemma as stated.

The following four lemmas hold for sections which a priori are known to be compactly contained in $\Omega$.

Lemma 2.2 (Engulfing property). There exists $\theta$ such that if $x \in S_{\phi}(y, t)$, then $S_{\phi}(y, t) \subseteq S_{\phi}(x, \theta t)$.

The following lemma is used for an iteration argument in Sections 5 and 6 .

Lemma 2.3. Suppose $\alpha<\beta$ and $x \in \Omega_{\alpha}$. Then $S_{\phi}\left(x, C_{0}(\beta-\alpha)^{\gamma}\right) \subseteq \Omega_{\beta}$ for some $C_{0}$ and $\gamma$ depending only on $\lambda$ and $\Lambda$.

Lemma 2.4. There exist constants $C_{1}$ and $C_{2}$ depending only on $n, \lambda$ and $\Lambda$ such that

$$
C_{1} t^{n / 2} \leq\left|S_{\phi}(x, t)\right| \leq C_{2} t^{n / 2} .
$$

Lemma 2.4 says that the measure of any section depends essentially on the parameter $t$ and is comparable to the measure of an Euclidean ball of radius $\sqrt{t}$. However, a section may look like an ellipsoid in which the ratio between the longest axes and the shortest axes goes to infinity as the parameter $t$ goes to 0 . In other words, the eccentricity of a section is not bounded by constants depending only on $\lambda, \Lambda$, and $n$.

The following lemma is a simple consequence of Lemma 2.4

Lemma 2.5. Given $0<\alpha<1$ there exists $0<\epsilon<1$ depending only on $\lambda, \Lambda$ and $n$ such that $(1-\epsilon) \mu\left(S_{\phi}(x, t)\right) \leq \mu\left(S_{\phi}(x, \alpha t)\right)$ for any section $S_{\phi}(x, t) \Subset \Omega$.

We will also need the following observation about normalization. Let $S_{\phi}(\bar{x}, t)$ be a section and let $T$ normalize $S_{\phi}(\bar{x}, t)$. Let $\tilde{\phi}(y)=\frac{1}{t} \phi\left(T^{-1} y\right)$. We then have $T\left(S_{\phi}(\bar{x}, t)\right)=S_{\tilde{\phi}}(T \bar{x}, 1)$ and $\operatorname{det} D^{2} \tilde{\phi}(y)=\frac{1}{t|\operatorname{det} T|^{\frac{2}{n}}} \operatorname{det} D^{2} \phi\left(T^{-1} y\right)$. Since $B_{\alpha_{n}}(0) \subseteq$ $T\left(S_{\phi}(\bar{x}, t)\right) \subseteq B_{1}(0)$, it follows that $\frac{C_{1}}{t} \leq|\operatorname{det} T|^{\frac{2}{n}} \leq \frac{C_{2}}{t}$, where $C_{1}$ and $C_{2}$ depend on $\lambda, \Lambda$ and $n$. Hence, $\lambda^{\prime} \leq \operatorname{det} D^{2} \tilde{\phi}(y) \leq \Lambda^{\prime}$ on $S_{\tilde{\phi}}(T \bar{x}, 1)$. Letting $\psi(y)=\tilde{\phi}(y)-$ $\tilde{\phi}(\bar{y})-\nabla \tilde{\phi}(\bar{y}) \cdot(y-\bar{y})-1$ where $T \bar{x}=\bar{y}$, we have $\psi=0$ on $\partial S_{\tilde{\phi}}(\bar{y}, 1)$ and $\lambda^{\prime} \leq$ $\operatorname{det} D^{2} \psi \leq \Lambda^{\prime}$. In particular, Lemma 2.1 holds for $\psi$.

Next, we state a covering lemma for sections, which is in [G01, Chapter 6].

Lemma 2.6. Let $A \subseteq \Omega$ and suppose that for each $x \in A$ a section $S_{\phi}(x, t) \Subset \Omega$ is given such that $t$ is bounded by a fixed number $\eta$. Then, there exists a countable subfamily $\left\{S_{\phi}\left(x_{k}, t_{k}\right)\right\}_{k}$ with the following properties:

(1) $A \subseteq \bigcup_{k} S_{\phi}\left(x_{k}, t_{k}\right)$, and

(2) for $0<\epsilon<\epsilon_{0}(n, \lambda, \Lambda)$ we have that the family $\left\{S_{\phi}\left(x_{k},(1-\epsilon) t_{k}\right)\right\}_{k}$ has bounded overlaps, more precisely,

$$
\sum_{k} \chi_{S_{\phi}\left(x_{k},(1-\epsilon) t_{k}\right)}(x) \leq C \log \frac{1}{\epsilon},
$$

where $C$ depends also on $n, \lambda$, and $\Lambda$, and $\chi_{E}$ denotes the characteristic function of the set $E$.

The next lemma is of fundamental importance in setting up an iteration argument, and it complements the previous lemma. Its proof is in [CG97. 
Lemma 2.7. Let $\mathcal{O} \subset \Omega$ and $0<\epsilon<1$. Suppose that for each $x \in \mathcal{O}$, there exists a section $S_{\phi}\left(x, t_{x}\right) \subset \Omega$ with $t_{x} \leq \beta$ and

$$
\frac{\mu\left(S_{\phi}\left(x, t_{x}\right) \cap \mathcal{O}\right)}{\mu\left(S_{\phi}\left(x, t_{x}\right)\right)}=\epsilon
$$

Then, there exists a countable subfamily of sections $\left\{S_{\phi}\left(x_{k}, t_{k}\right)\right\}_{k}$ and $0<\delta(\epsilon)<1$ such that $\mathcal{O} \subseteq \bigcup_{k} S_{\phi}\left(x_{k}, t_{k}\right)$ and $\mu(\mathcal{O}) \leq \delta(\epsilon) \mu\left(\bigcup_{k} S_{\phi}\left(x_{k}, t_{k}\right)\right)$.

We now define and state a theorem for the maximal function that will be used in Section 6 .

Definition 2.8. For $x \in \Omega_{\alpha_{0}}$, define

$$
\mathcal{M}_{\mu}(f)(x)=\sup _{t \leq \eta_{0} / 2} \frac{1}{\mu\left(S_{\phi}(x, t)\right)} \int_{S_{\phi}(x, t)}|f(y)| d \mu(y) .
$$

Theorem 2.9. There exists a constant $C$ depending on $\lambda$ and $\Lambda$ such that

$$
\mu\left(\left\{x \in \Omega_{\alpha_{0}}: \mathcal{M}_{\mu}(f)(x)>\beta\right\}\right) \leq \frac{C}{\beta} \int_{\Omega}|f(y)| d \mu(y) .
$$

Proof. Let $A_{\beta}=\left\{x \in \Omega_{\alpha_{0}}: M_{\mu}(f)(x)>\beta\right\}$. For each $x \in A_{\beta}$, there exists $t_{x} \leq \eta_{0} / 2$ such that

$$
\frac{1}{\mu\left(S_{\phi}\left(x, t_{x}\right)\right)} \int_{S_{\phi}\left(x, t_{x}\right)}|f(y)| d \mu(y) \geq \beta .
$$

Consider the family $S_{\phi}\left(x, 2 t_{x}\right)$ ). By Lemma 2.6 there exists a countable subfamily $\left\{S_{\phi}\left(x_{k}, 2 t_{k}\right)\right\}_{k}$ such that $A_{\beta} \subseteq \bigcup S_{\phi}\left(x_{k}, 2 t_{k}\right)$ and such that $\sum_{k} \chi_{s_{\phi}\left(x_{k},(1-\epsilon) 2 t_{k}\right)}(x)$ $\leq C \log \frac{1}{\epsilon}$ for every $\epsilon \leq \epsilon_{0}$.

We have

$$
\mu\left(A_{\beta}\right) \leq \sum_{k} \mu\left(S_{\phi}\left(x_{k}, 2 t_{k}\right)\right) \leq C \sum_{k} \mu\left(S_{\phi}\left(x_{k},(1-\epsilon) 2 t_{k}\right)\right) .
$$

Also,

$$
\begin{aligned}
\beta & \leq \frac{1}{\mu\left(S_{\phi}\left(x_{k}, t_{k}\right)\right)} \int_{S_{\phi}\left(x_{k}, t_{k}\right)}|f(y)| d \mu(y) \\
& \leq C \frac{1}{\mu\left(S_{\phi}\left(x_{k},(1-\epsilon) 2 t_{k}\right)\right)} \int_{S_{\phi}\left(x_{k},(1-\epsilon) 2 t_{k}\right)}|f(y)| d \mu(y) .
\end{aligned}
$$

Therefore,

$$
\beta \mu\left(S_{\phi}\left(x_{k},(1-\epsilon) 2 t_{k}\right)\right) \leq C \int_{S_{\phi}\left(x_{k},(1-\epsilon) 2 t_{k}\right)}|f(y)| d \mu(y) .
$$


Hence,

$$
\begin{aligned}
\mu\left(A_{\beta}\right) & \leq \frac{1}{\beta} C \sum_{k} \beta \mu\left(S_{\phi}\left(x_{k},(1-\epsilon) 2 t_{k}\right)\right) \leq \frac{C}{\beta} \sum_{k} \int_{S_{\phi}\left(x_{k},(1-\epsilon) 2 t_{k}\right)}|f(y)| d \mu(y) \\
& =\frac{C}{\beta} \sum_{k} \int_{\Omega} \chi_{S_{\phi}\left(x_{k},(1-\epsilon) 2 t_{k}\right)}(y)|f(y)| d \mu(y) \\
& =\frac{C}{\beta} \int_{\Omega} \sum_{k} \chi_{S_{\phi}\left(x_{k},(1-\epsilon) 2 t_{k}\right)}(y)|f(y)| d \mu(y) \\
& \leq \frac{C \log \frac{1}{\epsilon}}{\beta} \int_{\Omega}|f(y)| d \mu(y) .
\end{aligned}
$$

\section{MAIN DEFinitions}

In this section we define the quasidistance given by the sections of $\phi$, and using this quasidistance we define the sets where the solution $u$ is touched from above and below by certain polynomials. We also prove in this section a lemma for solutions to the Monge-Ampère equation which is the second step mentioned in the Introduction.

First, we begin with a simple lemma that is used repeatedly in our calculations.

Lemma 3.1. Let $\Omega$ be a bounded domain, $\phi \in C^{2}(\Omega)$ convex with $\operatorname{det} D^{2} \phi(x)>0$ for $x \in \Omega$, and let $u \in C^{2}(\Omega)$. Let $w=u+\phi$, let $\Gamma(w)$ be the convex envelope of $w$, and let the contact set $\mathcal{C}=\{x \in \Omega: w(x)=\Gamma(w)(x)\}$. Suppose $E \subset \mathcal{C}$ and $F \subset \Omega$ are measurable sets such that $\partial \phi(F) \subset \partial w(E)$.

Then

$$
M \phi(F) \leq \frac{1}{n^{n}} \int_{E}\left(\left(\frac{L_{\phi} u(x)}{\operatorname{det} D^{2} \phi(x)}+n\right)^{+}\right)^{n} \operatorname{det} D^{2} \phi(x) d x .
$$

Proof. We have

$$
\begin{aligned}
M \phi(F) & \leq M w(E) \leq \int_{E} \operatorname{det} D^{2} w(x) d x \\
& =\int_{E} \frac{\operatorname{det} \Phi(x) \operatorname{det} D^{2} w(x)}{\operatorname{det} \Phi(x)} d x \\
& \leq \frac{1}{n^{n}} \int_{E} \frac{1}{\operatorname{det} \Phi(x)}\left(\operatorname{trace}\left(\Phi(x) D^{2} w(x)\right)\right)^{n} d x \\
& =\frac{1}{n^{n}} \int_{E} \frac{1}{\left(\operatorname{det} D^{2} \phi(x)\right)^{n-1}}\left(\operatorname{trace}\left(\Phi(x)\left(D^{2} u(x)+D^{2} \phi(x)\right)\right)\right)^{n} d x \\
& =\frac{1}{n^{n}} \int_{E} \frac{1}{\left(\operatorname{det} D^{2} \phi(x)\right)^{n-1}}\left(L_{\phi} u(x)+n \operatorname{det} D^{2} \phi(x)\right)^{n} d x,
\end{aligned}
$$

and (3.1) follows.

3.1. A quasi-metric. Let $\Omega$ be a convex normalized domain in $\mathbb{R}^{n}, \phi \in C^{2}(\Omega)$ convex, $\phi=0$ on $\partial \Omega, 0<\lambda \leq \operatorname{det} D^{2} \phi \leq \Lambda$ in $\Omega$.

Definition 3.2. Let $S_{\phi}\left(x_{0}, \eta_{0}\right) \Subset \Omega$. Given $x \in S_{\phi}\left(x_{0}, \eta_{0}\right)$ we define

$$
d\left(x, x_{0}\right)^{2}=\inf \left\{t: x \in S_{\phi}\left(x_{0}, t\right)\right\} .
$$

Remark 3.3. If $\phi \in C^{1}(\Omega)$, then $d\left(x, x_{0}\right)^{2}=\phi(x)-\phi\left(x_{0}\right)-\nabla \phi\left(x_{0}\right) \cdot\left(x-x_{0}\right)$. 
Lemma 3.4. The function $d$ in Definition 3.2 satisfies:

(a) $d(x, y)^{2} \leq \theta\left(d(x, z)^{2}+d(y, z)^{2}\right)$ whenever all terms are defined.

(b) If $S_{\phi}\left(\bar{x}, \eta_{0}\right) \Subset \Omega$, then $d(x, \bar{x})^{2}$ is a convex function of $x$ on the set $S_{\phi}\left(\bar{x}, \eta_{0}\right)$.

Proof. (a) Let $x \in S_{\phi}\left(z, t_{1}\right), y \in S_{\phi}\left(z, t_{2}\right)$ and $t=\max \left\{t_{1}, t_{2}\right\}$. Since $y \in S_{\phi}(z, t)$, we have by the engulfing property $S_{\phi}(z, t) \subset S_{\phi}(y, \theta t)$, and $x \in S_{\phi}\left(z, t_{1}\right) \subset S_{\phi}(z, t) \subset$ $S_{\phi}(y, \theta t)$. Hence $d(x, y)^{2} \leq \theta t \leq \theta\left(t_{1}+t_{2}\right)$, and so

$$
\begin{aligned}
d(x, y)^{2} & \leq \theta \inf \left\{t_{1}+t_{2}: x \in S_{\phi}\left(z, t_{1}\right), y \in S_{\phi}\left(z, t_{2}\right)\right\} \\
& =\theta\left(\inf \left\{t_{1}: x \in S_{\phi}\left(z, t_{1}\right)\right\}+\inf \left\{t_{2}: y \in S_{\phi}\left(z, t_{2}\right)\right\}\right) \\
& =\theta\left(d(x, z)^{2}+d(y, z)^{2}\right) .
\end{aligned}
$$

Note that if $x, y \in S_{\phi}(z, \delta)$ and $\delta \theta \leq \eta_{0}$, then $d(x, y)^{2}$ is defined because $x \in$ $S_{\phi}(y, \theta \delta)$.

(b) Let $x_{1}, x_{2} \in S_{\phi}\left(\bar{x}, \eta_{0}\right)$ and $0<\lambda<1$. We have

$$
d\left(\lambda x_{1}+(1-\lambda) x_{2}, \bar{x}\right)^{2}=\inf \left\{t: \lambda x_{1}+(1-\lambda) x_{2} \in S_{\phi}(\bar{x}, t)\right\} .
$$

Suppose $x_{1} \in S_{\phi}\left(\bar{x}, t_{1}\right)$ and $x_{2} \in S_{\phi}\left(\bar{x}, t_{2}\right)$, and let $\psi(x)=\phi(x)-\phi(\bar{x})-\nabla \phi(\bar{x})$. $(x-\bar{x})$. The function $\psi$ is convex, and so

$$
\psi\left(\lambda x_{1}+(1-\lambda) x_{2}\right) \leq \lambda \psi\left(x_{1}\right)+(1-\lambda) \psi\left(x_{2}\right)<\lambda t_{1}+(1-\lambda) t_{2} .
$$

Then $\lambda x_{1}+(1-\lambda) x_{2} \in S_{\phi}\left(\bar{x}, \lambda t_{1}+(1-\lambda) t_{2}\right)$ and

$$
\inf \left\{t: \lambda x_{1}+(1-\lambda) x_{2} \in S_{\phi}(\bar{x}, t)\right\} \leq \lambda t_{1}+(1-\lambda) t_{2} .
$$

Since $t_{1}$ and $t_{2}$ are arbitrary, we get

$$
\begin{aligned}
& \inf \left\{t: \lambda x_{1}+(1-\lambda) x_{2} \in S_{\phi}(\bar{x}, t)\right\} \\
& \leq \lambda \inf \left\{t: x_{1} \in S_{\phi}(\bar{x}, t)\right\}+(1-\lambda) \inf \left\{t: x_{2} \in S_{\phi}(\bar{x}, t)\right\}
\end{aligned}
$$

that is,

$$
d\left(\lambda x_{1}+(1-\lambda) x_{2}, \bar{x}\right)^{2} \leq \lambda d\left(x_{1}, \bar{x}\right)^{2}+(1-\lambda) d\left(x_{2}, \bar{x}\right)^{2} .
$$

3.2. Spaces. For the following definitions we fix $0<\alpha_{0}<1$ once and for all.

Definition 3.5. Given $u \in C^{2}(\Omega), 0<\alpha_{0}<1, M>1, \theta$ the engulfing constant, and $\theta^{2} / M \leq \eta\left(\alpha_{0}\right)$, we define

$$
\begin{gathered}
G_{M}^{+}(u)=\left\{\bar{x} \in \Omega_{\alpha_{0}}: u(x) \leq u(\bar{x})+\nabla u(\bar{x}) \cdot(x-\bar{x})+M d(x, \bar{x})^{2},\right. \\
\left.\forall x \in S_{\phi}\left(\bar{x}, \theta^{2} / M\right)\right\}, \\
G_{M}^{-}(u)=\left\{\bar{x} \in \Omega_{\alpha_{0}}: u(x) \geq u(\bar{x})+\nabla u(\bar{x}) \cdot(x-\bar{x})-M d(x, \bar{x})^{2},\right. \\
\left.\forall x \in S_{\phi}\left(\bar{x}, \theta^{2} / M\right)\right\},
\end{gathered}
$$

and

$$
G_{M}(u)=G_{M}^{+}(u) \cap G_{M}^{-}(u)
$$




\subsection{A lemma for the Monge-Ampère equation.}

Lemma 3.6. Let $\Omega$ be a normalized convex domain, $\phi \in C^{2}(\Omega)$ with $\phi=0$ on $\partial \Omega$, and $0 \leq \operatorname{det} D^{2} \phi \leq \Lambda$ in $\Omega$. Let $0<\alpha_{0}<\alpha_{1}<1$ and

$$
\mathcal{G}_{M}^{+}(\phi)=\left\{\bar{x} \in \Omega_{\alpha_{0}}: \phi(x) \leq \phi(\bar{x})+\nabla \phi(\bar{x}) \cdot(x-\bar{x})+M|x-\bar{x}|^{2}, \quad \forall x \in \Omega_{\alpha_{1}}\right\} .
$$

Then

$$
\left|\Omega_{\alpha_{0}} \backslash \mathcal{G}_{M(t)}^{+}(\phi)\right| \leq\left(1-\frac{1}{(t+1)^{n}}\right)\left|\Omega_{\alpha_{0}}\right|
$$

where $M(t)=\left(\frac{t+1}{t}\right) \Lambda^{1 / n} C\left(\alpha_{1}\right)$ and for all $0<t<1$.

Proof. Let $w$ be the solution to $\operatorname{det} D^{2} w=1$ in $\Omega$ with $w=0$ on $\partial \Omega$. It follows from Pogorelov's estimate, G01, inequality (4.2.6)], that

$$
\mathcal{G}_{C\left(\alpha_{1}\right)}^{+}(w)=\Omega_{\alpha_{0}} .
$$

We have that $\operatorname{det} D^{2}\left(\Lambda^{1 / n} w\right)=\Lambda \geq \operatorname{det} D^{2} \phi$. Then by the comparison principle, G01, Theorem 1.4.6], $\Lambda^{1 / n} w \leq \phi$ in $\Omega$, and so $-w / 2 \geq-\phi /\left(2 \Lambda^{1 / n}\right) \geq 0$. Consequently, $\frac{w}{2} \geq w-\frac{\phi}{2 \Lambda^{1 / n}} \geq w$ in $\Omega$.

Let $v_{t}(x)=t\left(w(x)-\frac{\phi(x)}{2 \Lambda^{1 / n}}\right)+(1-t) \frac{w(x)}{2}$. Then

$$
w \leq w-\frac{\phi}{2 \Lambda^{1 / n}} \leq v_{t} \leq \frac{w}{2},
$$

for $0<t<1$. Let $\Gamma\left(v_{t}\right)$ be the convex envelope of $v_{t}$ in $\Omega$, and let $\mathcal{C}_{t}$ be the set of contact points, $\mathcal{C}_{t}=\left\{x \in \Omega: \Gamma\left(v_{t}\right)(x)=v_{t}(x)\right\}$. Then $w \leq \Gamma\left(v_{t}\right) \leq v_{t} \leq w / 2$, and so $\nabla(w / 2)(\Omega) \subset \partial \Gamma\left(v_{t}\right)(\Omega)$, by [G01, Lemma 1.4.1]. Therefore

$$
\begin{aligned}
|\nabla(w / 2)(\Omega)| & \leq\left|\partial \Gamma\left(v_{t}\right)(\Omega)\right|=\left|\partial \Gamma\left(v_{t}\right)\left(\mathcal{C}_{t}\right)\right| \quad \text { by Lemma 7.2 } \\
& \leq \int_{\mathcal{C}_{t}}\left(\left(\left(M\left(\frac{t+1}{2} w\right)\right)^{1 / n}-\left(M\left(\frac{t}{2 \Lambda^{1 / n}} \phi\right)\right)^{1 / n}\right)^{+}\right)^{n} d x \\
& \leq \int_{\mathcal{C}_{t}}\left(\frac{t+1}{2}-\frac{t}{2 \Lambda^{1 / n}}(M \phi)^{1 / n}\right)^{n} d x \\
& \leq\left|\mathcal{C}_{t}\right|\left(\frac{t+1}{2}\right)^{n}
\end{aligned}
$$

by Theorem 7.1 since $v_{t}(x)=\left(\frac{t+1}{2}\right) w(x)-t \frac{\phi(x)}{2 \Lambda^{1 / n}}$. Hence we obtain

$$
\frac{1}{(t+1)^{n}}|\Omega| \leq\left|\mathcal{C}_{t}\right| \text {. }
$$

This implies $\left|\Omega_{\alpha_{0}} \backslash \mathcal{C}_{t}\right| \leq\left(1-\frac{1}{(t+1)^{n}}\right)\left|\Omega_{\alpha_{0}}\right|$, and since $\mathcal{C}_{t} \cap \Omega_{\alpha_{0}} \subset \Omega_{\alpha_{0}} \cap \mathcal{G}_{M(t)}^{+}(\phi)$, we obtain the desired estimate.

Notice that if $0<\alpha_{0}<\alpha_{1}<1$, then $\operatorname{dist}\left(\Omega_{\alpha_{0}}, \partial \Omega_{\alpha_{1}}\right) \leq C\left(\alpha_{0}, \alpha_{1}\right)$. Hence if $\bar{x} \in \Omega_{\alpha_{0}}$, then the Euclidean ball $B_{\sqrt{t} / \beta}(\bar{x}) \subset \Omega_{\alpha_{1}}$ for $t \leq\left(\beta C\left(\alpha_{0}, \alpha_{1}\right)\right)^{2}$.

Lemma 3.7. Let

$$
A_{\beta}=\left\{x \in \Omega_{\alpha_{0}}: B_{\sqrt{t} / \beta}(x) \subset S_{\phi}(x, t), \quad \forall t \leq \eta_{0}\right\},
$$

where $\eta_{0} \leq \min \left\{\eta\left(\alpha_{0}\right),\left(\beta C\left(\alpha_{0}, \alpha_{1}\right)\right)^{2}\right\}$. Then we have

$$
\mathcal{G}_{\beta^{2}}^{+}(\phi) \subset A_{\beta} .
$$


Proof. Let $\bar{x} \in \mathcal{G}_{\beta^{2}}^{+}(\phi)$, and let $x \in B_{\sqrt{t} / \beta}(\bar{x})$. We have $\phi(x) \leq \phi(\bar{x})+\nabla \phi(\bar{x})$. $(x-\bar{x})+\beta^{2}|x-\bar{x}|^{2}$ for each $x \in \Omega_{\alpha_{1}}$. By the choice of $\eta_{0}$, we have $B_{\sqrt{t} / \beta}(\bar{x}) \subset \Omega_{\alpha_{1}}$. Then $\phi(x)<\phi(\bar{x})+\nabla \phi(\bar{x}) \cdot(x-\bar{x})+t$, that is, $x \in S_{\phi}(\bar{x}, t)$ and so $\bar{x} \in A_{\beta}$.

Remark 3.8. From Lemma 3.7 $\Omega_{\alpha_{0}} \backslash A_{\beta} \subset \Omega_{\alpha_{0}} \backslash \mathcal{G}_{\beta^{2}}^{+}(\phi)$, and therefore

$$
\mu\left(\Omega_{\alpha_{0}} \backslash A_{\beta}\right) \leq \mu\left(\Omega_{\alpha_{0}} \backslash \mathcal{G}_{\beta^{2}}^{+}(\phi)\right) \leq \Lambda\left|\Omega_{\alpha_{0}} \backslash \mathcal{G}_{\beta^{2}}^{+}(\phi)\right| \leq \frac{C\left(n, \Lambda, \alpha_{0}\right)}{\beta^{2}},
$$

by Lemma 3.6 ,

Remark 3.9. If $\bar{x} \in A_{\beta}$, then $d(x, \bar{x})^{2} \leq \beta^{2}|x-\bar{x}|^{2}$ for all $x \in S_{\phi}\left(\bar{x}, \eta_{0}\right)$.

Indeed, we have $B_{\sqrt{t} / \beta}(\bar{x}) \subset S_{\phi}(\bar{x}, t)$ for all $t \leq \eta_{0}$. If $x \in S_{\phi}\left(\bar{x}, \eta_{0}\right)$, then

$$
\left\{t: x \in B_{\sqrt{t} / \beta}(\bar{x})\right\} \subset\left\{t: x \in S_{\phi}(\bar{x}, t)\right\},
$$

and so, $\inf \left\{t: x \in S_{\phi}(\bar{x}, t)\right\} \leq \inf \left\{t: x \in B_{\sqrt{t} / \beta}(\bar{x})\right\}$. Consequently, $d(x, \bar{x})^{2} \leq$ $\beta^{2}|x-\bar{x}|^{2}$.

Remark 3.10. We have

$$
A_{\beta} \cap G_{\beta}(u) \subset\left\{x \in \Omega_{\alpha_{0}}:\left|D_{i j} u(x)\right| \leq \beta^{3}, \text { for } i, j=1, \cdots, n\right\}
$$

for each $\beta$ such that $\theta^{2} / \beta \leq \eta\left(\alpha_{0}\right)$.

In fact, if $\bar{x} \in A_{\beta} \cap G_{\beta}(u)$, then

$$
-\beta d(x, \bar{x})^{2} \leq u(x)-u(\bar{x})-\nabla u(\bar{x}) \cdot(x-\bar{x}) \leq \beta d(x, \bar{x})^{2}
$$

for all $x \in S_{\phi}\left(\bar{x}, \theta^{2} / \beta\right)$. Since $\bar{x} \in A_{\beta}$, by the previous remark $d(x, \bar{x})^{2} \leq \beta^{2}|x-\bar{x}|^{2}$ we get

$$
-\beta^{3}|x-\bar{x}|^{2} \leq u(x)-u(\bar{x})-\nabla u(\bar{x}) \cdot(x-\bar{x}) \leq \beta^{3}|x-\bar{x}|^{2}
$$

for each $x \in S_{\phi}\left(\bar{x}, \theta^{2} / \beta\right)$, and so $\left|D_{i j} u(\bar{x})\right| \leq \beta^{3}$.

If $0<\alpha \leq \alpha_{0}$, then from Remark 3.10 we have that

$$
\begin{aligned}
\left\{x \in \Omega_{\alpha}:\left|D_{i j} u(x)\right|>\beta^{3}\right\} & =\Omega_{\alpha} \backslash\left\{x \in \Omega_{\alpha}:\left|D_{i j} u(x)\right| \leq \beta^{3}\right\} \\
& \subset \Omega_{\alpha} \backslash\left(A_{\beta} \cap G_{\beta}(u)\right), \quad \text { where } \theta^{2} / \beta \leq \eta\left(\alpha_{0}\right) \\
& \subset\left(\Omega_{\alpha} \backslash A_{\beta}\right) \cup\left(\Omega_{\alpha} \backslash G_{\beta}(u)\right) .
\end{aligned}
$$

\section{Density Lemmas}

In this section we prove two density lemmas that will be used in Sections 5 and 6 to prove the decay of $\mu\left(\Omega_{\alpha} \backslash G_{\beta}(u)\right)$ for $\beta$ large.

Lemma 4.1. Let $\Omega$ be a normalized convex domain, $\phi \in C^{2}(\Omega), \phi=0$ on $\partial \Omega$, and $\lambda \leq \operatorname{det} D^{2} \phi \leq \Lambda$. Suppose $u \in C^{2}(\Omega), 0 \leq u \leq 1$, and $L_{\phi} u=0$ in $\Omega$.

Then for each $\epsilon>0$ there exists $\delta(\epsilon)>0$ such that if $M \geq \frac{1}{\delta(\epsilon)}$, then we have

$$
\mu\left(G_{M / t_{0}}^{-}(u) \cap S_{\phi}\left(x_{0}, t_{0}\right)\right)>(1-\epsilon) \mu\left(S_{\phi}\left(x_{0}, t_{0}\right)\right)
$$

for all sections $S_{\phi}\left(x_{0}, t_{0}\right) \subset \Omega_{\alpha_{0}}$ with $t_{0} \leq \eta_{0} / \theta$. 
Proof. Let $T$ normalize the section $S_{\phi}\left(x_{0}, t_{0}\right)$. Let

$$
\begin{aligned}
\tilde{\phi}(y) & =\frac{1}{t_{0}} \phi\left(T^{-1} y\right), \quad y_{0}=T x_{0}, \\
\psi(y) & =\tilde{\phi}(y)-\tilde{\phi}\left(y_{0}\right)-\nabla \tilde{\phi}\left(y_{0}\right) \cdot\left(y-y_{0}\right)-1, \\
\text { and } \quad \tilde{u}(y) & =u\left(T^{-1} y\right) .
\end{aligned}
$$

We have $T\left(S_{\phi}\left(x_{0}, t_{0}\right)\right)=S_{\tilde{\phi}}\left(y_{0}, 1\right)=\tilde{\Omega}$ is normalized, $\psi=0$ on $\partial \tilde{\Omega}$, and $\lambda^{\prime} \leq$ $\operatorname{det} D^{2} \psi \leq \Lambda^{\prime}$ in $\tilde{\Omega}$ by the observation after Lemma 2.5 . For each $0<\alpha<1$ there exists $\eta(\alpha)$ such that if $\bar{y} \in \tilde{\Omega}_{\alpha}$, then $S_{\psi}(\bar{y}, \eta(\alpha)) \subset \subset \tilde{\Omega}$ by Lemma 2.1. Therefore if $\bar{y} \in \tilde{\Omega}_{\alpha}$, then $\psi(\bar{y})+\nabla \psi(\bar{y}) \cdot(y-\bar{y})+\eta(\alpha)<0$ for all $y \in \overline{\tilde{\Omega}}$.

Let $w_{\alpha}(y)=\eta(\alpha) \tilde{u}(y)+\psi(y)$, and let $\gamma_{\alpha}$ be the convex envelope of $w_{\alpha}$ in $\tilde{\Omega}$, and

$\mathcal{C}_{\alpha}=\left\{y \in \tilde{\Omega}: w_{\alpha}(y)=\gamma_{\alpha}(y)\right.$, and $\exists \ell_{y}$ supporting hyperplane

$$
\text { to } \left.\gamma_{\alpha} \text { at } y \text { with } \ell_{y}<0 \text { in } \tilde{\Omega}\right\} \text {. }
$$

Claim 1. $\nabla \psi\left(\tilde{\Omega}_{\alpha}\right) \subset \nabla w_{\alpha}\left(\mathcal{C}_{\alpha}\right)$.

To prove this claim, let $\bar{y} \in \tilde{\Omega}_{\alpha}$ and

$$
\begin{aligned}
\delta & =\max \left\{\psi(\bar{y})+\nabla \psi(\bar{y}) \cdot(y-\bar{y})+\eta(\alpha)-w_{\alpha}(y): y \in \overline{\widetilde{\Omega}}\right\} \\
& =\psi(\bar{y})+\nabla \psi(\bar{y}) \cdot\left(y^{*}-\bar{y}\right)+\eta(\alpha)-w_{\alpha}\left(y^{*}\right),
\end{aligned}
$$

for some $y^{*} \in \overline{\tilde{\Omega}}$. Note that $y^{*} \in \tilde{\Omega}$. Because since $\bar{y} \in \overline{\tilde{\Omega}}$, we have $\delta \geq \psi(\bar{y})+\eta(\alpha)-$ $w_{\alpha}(\bar{y})=\eta(\alpha)(1-\tilde{u}(\bar{y})) \geq 0$. Also, if $y^{*} \in \partial \tilde{\Omega}$, then we would have $\delta<-w_{\alpha}\left(y^{*}\right) \leq 0$, which is contradictory. Therefore

$\psi(\bar{y})+\nabla \psi(\bar{y}) \cdot\left(y^{*}-\bar{y}\right)+\eta(\alpha)-w_{\alpha}\left(y^{*}\right) \geq \psi(\bar{y})+\nabla \psi(\bar{y}) \cdot(y-\bar{y})+\eta(\alpha)-w_{\alpha}(y)$ for all $y \in \tilde{\Omega}$, that is,

$$
w_{\alpha}(y) \geq w_{\alpha}\left(y^{*}\right)+\nabla \psi(\bar{y}) \cdot\left(y-y^{*}\right)
$$

for all $y \in \tilde{\Omega}$. Since $w_{\alpha}\left(y^{*}\right)+\nabla \psi(\bar{y}) \cdot\left(y-y^{*}\right)$ is convex as a function of $y$, we have that

$$
w_{\alpha}(y) \geq \gamma_{\alpha}(y) \geq w_{\alpha}\left(y^{*}\right)+\nabla \psi(\bar{y}) \cdot\left(y-y^{*}\right),
$$

and so $w_{\alpha}\left(y^{*}\right)=\gamma_{\alpha}\left(y^{*}\right)$. Also $w_{\alpha}\left(y^{*}\right)+\nabla \psi(\bar{y}) \cdot\left(y-y^{*}\right)=\psi(\bar{y})+\nabla \psi(\bar{y}) \cdot(y-\bar{y})+$ $\eta(\alpha)-\delta<-\delta \leq 0$ in $\tilde{\Omega}$, and therefore $\nabla \psi(\bar{y}) \in \nabla w_{\alpha}\left(\mathcal{C}_{\alpha}\right)$. This proves Claim 1.

We have $D^{2} \tilde{\tilde{\phi}}(y)=\frac{1}{t_{0}}\left(T^{-1}\right)^{t} D^{2} \phi\left(T^{-1} y\right) T^{-1}$, so

$$
\left(D^{2} \tilde{\phi}(y)\right)^{-1}=t_{0} T\left(D^{2} \phi\left(T^{-1} y\right)\right)^{-1} T^{t} .
$$

Also $D^{2} \tilde{u}(y)=\left(T^{-1}\right)^{t} D^{2} u\left(T^{-1} y\right) T^{-1}$. If $\tilde{\Phi}(y)$ is the matrix of cofactors of $D^{2} \tilde{\phi}(y)$, i.e., $\tilde{\Phi}(y)=\left(D^{2} \tilde{\phi}(y)\right)^{-1} \operatorname{det} D^{2} \tilde{\phi}(y)$, then

$$
L_{\tilde{\phi}} \tilde{u}(y)=\operatorname{trace}\left(\tilde{\Phi}(y) D^{2} \tilde{u}(y)\right)=0
$$

in $\tilde{\Omega}$. Applying Lemma 3.1 with $u \rightsquigarrow \eta(\alpha) \tilde{u}, \phi \rightsquigarrow \tilde{\psi}, E=\mathcal{C}_{\alpha}, F=\tilde{\Omega}_{\alpha}$, we get

$$
\int_{\tilde{\Omega}_{\alpha}} \operatorname{det} D^{2} \tilde{\phi}(y) d y \leq \int_{\mathcal{C}_{\alpha}} \operatorname{det} D^{2} \tilde{\phi}(y) d y \text {. }
$$

We also have

$$
\operatorname{det} D^{2} \tilde{\phi}(y)=\frac{1}{t_{0}^{n}|\operatorname{det} T|^{2}} \operatorname{det} D^{2} \phi\left(T^{-1} y\right)
$$


and hence

$$
\int_{\tilde{\Omega}_{\alpha}} \operatorname{det} D^{2} \phi\left(T^{-1} y\right) d y \leq \int_{\mathcal{C}_{\alpha}} \operatorname{det} D^{2} \phi\left(T^{-1} y\right) d y
$$

We have $\tilde{\Omega}_{\alpha}=T\left(S_{\phi}\left(x_{0}, \alpha t_{0}\right)\right)$ and for now suppose

$$
\mathcal{C}_{\alpha} \subset T(A)
$$

where $A$ is a set that will be determined. Then

$$
\int_{S_{\phi}\left(x_{0}, \alpha t_{0}\right)} \operatorname{det} D^{2} \phi(x) d x \leq \int_{A} \operatorname{det} D^{2} \phi(x) d x,
$$

that is,

$$
\mu\left(S_{\phi}\left(x_{0}, \alpha t_{0}\right)\right) \leq \mu(A) .
$$

Claim 2. $\mathcal{C}_{\alpha} \subset T\left(S_{\phi}\left(x_{0}, t_{0}\right) \cap G_{1 /\left(\eta(\alpha) t_{0}\right)}^{-}(u)\right)$ for all $\alpha$ sufficiently close to one.

Proof of Claim 2. Let $\bar{y} \in \mathcal{C}_{\alpha}$. Then $\bar{y}=T \bar{x}$ for some $\bar{x} \in S_{\phi}\left(x_{0}, t_{0}\right)$ and

$$
\eta(\alpha) \tilde{u}(y)+\psi(y) \geq \ell(y) \text { for all } y \in T\left(S_{\phi}\left(x_{0}, t_{0}\right)\right)
$$

with equality at $y=\bar{y}$, for some $\ell$ affine with $\ell<0$ in $T\left(S_{\phi}\left(x_{0}, t_{0}\right)\right)$. We now claim that

$$
\phi(x) \leq \phi(\bar{x})+\nabla \phi(\bar{x}) \cdot(x-\bar{x})+d(x, \bar{x})^{2}
$$

for all $x \in S_{\phi}\left(\bar{x}, \eta_{0}\right)$. Indeed, let $x \in S_{\phi}(\bar{x}, t)$. Then $\phi(x)-(\phi(\bar{x})+\nabla \phi(\bar{x}) \cdot(x-\bar{x})) \leq t$, and hence

$$
\phi(x)-(\phi(\bar{x})+\nabla \phi(\bar{x}) \cdot(x-\bar{x})) \leq \inf \left\{t: x \in S_{\phi}(\bar{x}, t)\right\}=d(x, \bar{x})^{2} .
$$

This proves (4.4).

Recall that $\tilde{\phi}(y)=\frac{1}{t_{0}} \phi\left(T^{-1} y\right)$, where $T$ normalizes $S_{\phi}\left(x_{0}, t_{0}\right)$. Also $S_{\phi}\left(x_{0}, t_{0}\right) \subset$ $\Omega_{\alpha_{0}}$ and $\bar{x} \in S_{\phi}\left(x_{0}, t_{0}\right)$, so $\bar{x} \in \Omega_{\alpha_{0}}$, and $S_{\phi}\left(\bar{x}, \eta_{0}\right) \Subset \Omega$. Also from (4.4), if $x \in S_{\phi}\left(\bar{x}, \eta_{0}\right)$, then $\phi(x) \leq \phi(\bar{x})+\nabla \phi(\bar{x}) \cdot(x-\bar{x})+d(x, \bar{x})^{2}$. Consequently,

$$
\tilde{\phi}(y) \leq \tilde{\phi}(\bar{y})+\nabla \tilde{\phi}(\bar{y}) \cdot(y-\bar{y})+\frac{1}{t_{0}} d\left(T^{-1} y, T^{-1} \bar{y}\right)^{2},
$$

for all $y \in T\left(S_{\phi}\left(\bar{x}, \eta_{0}\right)\right)$. On the other hand, by definition of $\tilde{\phi}$ and $\psi$, the inequality (4.5) is equivalent to

$$
\psi(y) \leq \psi(\bar{y})+\nabla \psi(\bar{y}) \cdot(y-\bar{y})+\frac{1}{t_{0}} d\left(T^{-1} y, T^{-1} \bar{y}\right)^{2}=\tilde{\ell}_{\bar{y}}(y)+\frac{1}{t_{0}} d\left(T^{-1} y, T^{-1} \bar{y}\right)^{2}
$$

for all $y \in T\left(S_{\phi}\left(\bar{x}, \eta_{0}\right)\right)$, with equality at $\bar{y}=T \bar{x}$. Note that by engulfing and since $t_{0} \leq \eta_{0} / \theta$, we have $S_{\phi}\left(x_{0}, t_{0}\right) \subset S_{\phi}\left(\bar{x}, \theta t_{0}\right) \subset S_{\phi}\left(\bar{x}, \eta_{0}\right)$, and hence by (4.3) we get

$$
\eta(\alpha) \tilde{u}(y) \geq \ell(y)-\tilde{\ell}_{\bar{y}}(y)-\frac{1}{t_{0}} d\left(T^{-1} y, T^{-1} \bar{y}\right)^{2}
$$

for all $y \in T\left(S_{\phi}\left(x_{0}, t_{0}\right)\right)$ with equality for $y=\bar{y}$. Let

$$
g(y)=\ell(y)-\tilde{\ell}_{\bar{y}}(y)-\frac{1}{t_{0}} d\left(T^{-1} y, T^{-1} \bar{y}\right)^{2}
$$

for $y \in T\left(S_{\phi}\left(\bar{x}, \eta_{0}\right)\right)$, and

$$
B=\left\{y \in T\left(S_{\phi}\left(\bar{x}, \eta_{0}\right)\right): g(y) \geq 0\right\} .
$$

If $y \in \partial T\left(S_{\phi}\left(x_{0}, t_{0}\right)\right)$, then $\ell(y)<0$ and so $g(y)<-\tilde{\ell}_{\bar{y}}(y)-\frac{1}{t_{0}} d\left(T^{-1} y, T^{-1} \bar{y}\right)^{2} \leq$ $-\psi(y)=0$, and therefore $B \cap \partial T\left(S_{\phi}\left(x_{0}, t_{0}\right)\right)=\emptyset$. Also since, by Lemma 3.4(b), $g$ 
is concave, it follows that $B$ is connected. Hence $B \subset T\left(S_{\phi}\left(x_{0}, t_{0}\right)\right)$. This implies that

$$
\eta(\alpha) \tilde{u}(y) \geq g(y)
$$

for each $y \in T\left(S_{\phi}\left(\bar{x}, \eta_{0}\right)\right)$ with equality at $y=\bar{y}$. Because we have that $\eta(\alpha) \tilde{u}(y) \geq$ $g(y)$ for each $y \in T\left(S_{\phi}\left(x_{0}, t_{0}\right)\right)$, and if $y \in T\left(S_{\phi}\left(\bar{x}, \eta_{0}\right)\right) \backslash T\left(S_{\phi}\left(x_{0}, t_{0}\right)\right)$, then $y \notin B$ and so $g(y) \leq 0 \leq \eta(\alpha) \tilde{u}(y)$, that is, (4.6) follows. Since $\ell$ is a supporting hyperplane to $\eta(\alpha) \tilde{u}(y)+\psi(y)$ at $\bar{y}$, we then get

$$
\tilde{u}(y) \geq \tilde{u}(\bar{y})+\nabla \tilde{u}(\bar{y}) \cdot(y-\bar{y})-\frac{1}{t_{0} \eta(\alpha)} d\left(T^{-1} y, T^{-1} \bar{y}\right)^{2}
$$

for all $y \in T\left(S_{\phi}\left(\bar{x}, \eta_{0}\right)\right)$.

Hence we have proved that for each $0<\alpha<1$ we have

$$
\begin{gathered}
\mathcal{C}_{\alpha} \subset\left\{\bar{y} \in T\left(S_{\phi}\left(x_{0}, t_{0}\right)\right): \tilde{u}(y) \geq \tilde{u}(\bar{y})+\nabla \tilde{u}(\bar{y}) \cdot(y-\bar{y})-\frac{1}{t_{0} \eta(\alpha)} d\left(T^{-1} y, T^{-1} \bar{y}\right)^{2},\right. \\
\left.\forall y \in T\left(S_{\phi}\left(\bar{x}, \eta_{0}\right)\right) \text {, where } \bar{y}=T \bar{x}\right\} .
\end{gathered}
$$

Since $\tilde{u}(y)=u\left(T^{-1} y\right)$, it follows that

$$
\mathcal{C}_{\alpha} \subset T\left(S_{\phi}\left(x_{0}, t_{0}\right) \cap G_{1 / t_{0} \eta(\alpha)}^{-}(u)\right),
$$

as long as $t_{0} \eta(\alpha) \theta^{2} \leq \eta_{0}$ (as required to define the set $G$ with Definition 3.5) and for $t_{0} \theta \leq \eta_{0}$. Note that since $\eta(\alpha) \rightarrow 0$ as $\alpha \rightarrow 1$, the condition $t_{0} \eta(\alpha) \theta^{2} \leq \eta_{0}$ is always satisfied for $\alpha$ close to one. This completes the proof of Claim 2.

To complete the proof of the lemma, we note that from Lemma 2.5, given $\epsilon>0$ there exists $\alpha=\alpha(\epsilon)$ sufficiently close to one such that

$$
(1-\epsilon) \mu\left(S_{\phi}\left(x_{0}, t_{0}\right)\right) \leq \mu\left(S_{\phi}\left(x_{0}, \alpha t_{0}\right)\right)
$$

for all sections $S_{\phi}\left(x_{0}, t_{0}\right)$ compactly contained in $\Omega$. This inequality combined with (4.2) and Claim 2 yields the desired result with $M \geq \frac{1}{\delta(\epsilon)}=\frac{1}{\eta(\alpha(\epsilon))}$.

Lemma 4.2. Let $\Omega$ be a normalized convex domain, $\phi \in C^{2}(\Omega), \phi=0$ on $\partial \Omega$, and $\lambda \leq \operatorname{det} D^{2} \phi \leq \Lambda$. Suppose $u \in C^{2}(\Omega)$, and $L_{\phi} u=0$ in $\Omega$.

Then for each $\epsilon>0$ there exists $\delta(\epsilon)>0$ such that if $M \geq \frac{1}{\delta(\epsilon)}$ and $S_{\phi}\left(x_{0}, t_{0}\right) \cap$ $G_{\lambda}(u) \neq \emptyset$, then we have

$$
\mu\left(G_{\lambda M}^{-}(u) \cap S_{\phi}\left(x_{0}, t_{0}\right)\right)>(1-\epsilon) \mu\left(S_{\phi}\left(x_{0}, t_{0}\right)\right),
$$

where $t_{0}<1 / \lambda, S_{\phi}\left(x_{0}, t_{0}\right) \subset \Omega_{\alpha_{0}}$, and $\theta^{3} / \lambda \leq \eta_{0}$.

Proof. Let $S_{\phi}\left(x_{0}, t_{0}\right) \subset \Omega_{\alpha_{0}}, t_{0}<\frac{1}{\lambda} \leq \frac{\eta_{0}}{\theta^{3}}$, and let $\bar{x} \in S_{\phi}\left(x_{0}, t_{0}\right) \cap G_{\lambda}(u)$. Let $T$ normalize $S_{\phi}\left(x_{0}, t_{0}\right)$, and set

$$
\begin{aligned}
\tilde{\phi}(y) & =\frac{1}{t_{0}} \phi\left(T^{-1} y\right), \quad y_{0}=T x_{0}, \\
\psi(y) & =\tilde{\phi}(y)-\tilde{\phi}\left(y_{0}\right)-\nabla \tilde{\phi}\left(y_{0}\right) \cdot\left(y-y_{0}\right)-1, \\
\text { and } \quad \tilde{u}(y) & =\frac{1}{t_{0}} u\left(T^{-1} y\right) .
\end{aligned}
$$

We have as in Lemma 4.1 that $T\left(S_{\phi}\left(x_{0}, t_{0}\right)\right)=S_{\tilde{\phi}}\left(y_{0}, 1\right)=\tilde{\Omega}$ is normalized, $\psi=0$ on $\partial \tilde{\Omega}$, and $\lambda^{\prime} \leq \operatorname{det} D^{2} \psi \leq \Lambda^{\prime}$ in $\tilde{\Omega}$. 
Let $\bar{y}=T \bar{x}$. Since $\bar{x} \in S_{\phi}\left(x_{0}, t_{0}\right) \cap G_{\lambda}(u)$, we have

$$
-\lambda d(x, \bar{x})^{2} \leq u(x)-u(\bar{x})-\nabla u(\bar{x}) \cdot(x-\bar{x}) \leq \lambda d(x, \bar{x})^{2}
$$

for all $x \in S_{\phi}\left(\bar{x}, \theta^{2} / \lambda\right)$. Hence changing variables we get

$$
-\lambda \frac{d\left(T^{-1} y, T^{-1} \bar{y}\right)^{2}}{t_{0}} \leq \tilde{u}(y)-\tilde{u}(\bar{y})-\nabla \tilde{u}(\bar{y}) \cdot(y-\bar{y}) \leq \lambda \frac{d\left(T^{-1} y, T^{-1} \bar{y}\right)^{2}}{t_{0}}
$$

for all $y \in T\left(S_{\phi}\left(\bar{x}, \theta^{2} / \lambda\right)\right)$.

Since $\bar{x} \in S_{\phi}\left(x_{0}, t_{0}\right)$, we have $S_{\phi}\left(x_{0}, t_{0}\right) \subset S_{\phi}\left(\bar{x}, \theta t_{0}\right)$ by the engulfing property. So, if $x \in S_{\phi}\left(x_{0}, t_{0}\right)$, then $d(x, \bar{x})^{2} \leq \theta t_{0}$, and consequently $d\left(T^{-1} y, T^{-1} \bar{y}\right)^{2} \leq \theta t_{0}$ for all $y \in T\left(S_{\phi}\left(x_{0}, t_{0}\right)\right)$. Also since $t_{0}<1 / \lambda$, we have $S_{\phi}\left(x_{0}, t_{0}\right) \subset S_{\phi}\left(\bar{x}, \theta^{2} / \lambda\right)$. Then

and if we set

$$
-\lambda \theta \leq \tilde{u}(y)-\tilde{u}(\bar{y})-\nabla \tilde{u}(\bar{y}) \cdot(y-\bar{y}) \leq \lambda \theta,
$$

$$
v(y)=\frac{\tilde{u}(y)-\tilde{u}(\bar{y})-\nabla \tilde{u}(\bar{y}) \cdot(y-\bar{y})+\lambda \theta}{2 \lambda \theta},
$$

then $0 \leq v \leq 1$ in $\tilde{\Omega}$.

Let $0<\alpha<1$. There exist $1 \eta(\alpha)>0$ such that if $\bar{y} \in \tilde{\Omega}_{\alpha}$, then

$$
\psi(\bar{y})+\nabla \psi(\bar{y}) \cdot(y-\bar{y})+\eta(\alpha)(\theta+1 / 2)<0
$$

for all $y \in \overline{\tilde{\Omega}}$.

Define

$$
w_{\alpha}(y)=\eta(\alpha) v(y)+\psi(y) .
$$

We have $\psi \leq w_{\alpha} \leq \eta(\alpha)+\psi$ in $\tilde{\Omega}$. Let $\gamma_{\alpha}$ be the convex envelope of $w_{\alpha}$ in $\tilde{\Omega}$, and

$$
\mathcal{C}_{\alpha}=\left\{\bar{y} \in \tilde{\Omega}: w_{\alpha}(\bar{y})=\gamma_{\alpha}(\bar{y}), \quad \text { and } \exists \ell_{\bar{y}} \text { supporting hyperplane to } \gamma_{\alpha} \text { at } \bar{y},\right.
$$
with $\ell_{\bar{y}}<-\eta(\alpha)(\theta-1 / 2)$ in $\left.\tilde{\Omega}\right\}$.

Claim 1. $\nabla \psi\left(\tilde{\Omega}_{\alpha}\right) \subset \nabla w_{\alpha}\left(\mathcal{C}_{\alpha}\right)$.

Proof of Claim 1. Let $\bar{y} \in \tilde{\Omega}_{\alpha}$, and

$$
\delta=\max \left\{\psi(\bar{y})+\nabla \psi(\bar{y}) \cdot(y-\bar{y})+\eta(\alpha)-w_{\alpha}(y): y \in \bar{\Omega}\right\} .
$$

We have $\delta \geq \psi(\bar{y})-w_{\alpha}(\bar{y})=\eta(\alpha)(1-v(\bar{y})) \geq 0$, and if $y \in \partial \tilde{\Omega}$, then we have

$$
\psi(\bar{y})+\nabla \psi(\bar{y}) \cdot(y-\bar{y})+\eta(\alpha)-w_{\alpha}(y)<0 .
$$

Therefore there exists $y^{*} \in \tilde{\Omega}$ such that

$$
\begin{gathered}
\delta=\psi(\bar{y})+\nabla \psi(\bar{y}) \cdot\left(y^{*}-\bar{y}\right)+\eta(\alpha)-w_{\alpha}\left(y^{*}\right) \\
\geq \psi(\bar{y})+\nabla \psi(\bar{y}) \cdot(y-\bar{y})+\eta(\alpha)-w_{\alpha}(y)
\end{gathered}
$$

for all $y \in \tilde{\Omega}$. Hence

$$
w_{\alpha}(y) \geq w_{\alpha}\left(y^{*}\right)+\nabla \psi(\bar{y}) \cdot\left(y-y^{*}\right)
$$

for all $y \in \tilde{\Omega}$. Also note that

$$
\begin{aligned}
w_{\alpha}\left(y^{*}\right)+\nabla \psi(\bar{y}) \cdot\left(y-y^{*}\right) & =\psi(\bar{y})+\nabla \psi(\bar{y}) \cdot(y-\bar{y})+\eta(\alpha)-\delta \\
& \leq \psi(\bar{y})+\nabla \psi(\bar{y}) \cdot(y-\bar{y})+\eta(\alpha)<-\eta(\alpha)(\theta-1 / 2)
\end{aligned}
$$

for all $y \in \tilde{\Omega}$. This proves Claim 1 .

\footnotetext{
${ }^{1}$ Note that this $\eta(\alpha)$ is the $\eta(\alpha)$ from Lemma 2.1 divided by $\theta+\frac{1}{2}$.
} 
Proceeding as in Lemma 4.1, we have

$$
\int_{\tilde{\Omega}_{\alpha}} \operatorname{det} D^{2} \phi\left(T^{-1} y\right) d y \leq \int_{\mathcal{C}_{\alpha}} \operatorname{det} D^{2} \phi\left(T^{-1} y\right) d y .
$$

We have $\tilde{\Omega}_{\alpha}=T\left(S_{\phi}\left(x_{0}, \alpha t_{0}\right)\right)$. If we assume that

$$
\mathcal{C}_{\alpha} \subset T(A),
$$

where $A$ is a set to be determined, then we have

$$
\int_{S_{\phi}\left(x_{0}, \alpha t_{0}\right)} \operatorname{det} D^{2} \phi(x) d x \leq \int_{A} \operatorname{det} D^{2} \phi(x) d x,
$$

that is,

$$
\mu\left(S_{\phi}\left(x_{0}, \alpha t_{0}\right)\right) \leq \mu(A) .
$$

We next determine $A$.

Claim 2. The inequality (4.9) holds with $A=S_{\phi}\left(x_{0}, t_{0}\right) \cap G_{\lambda M}^{-}(u)$, for $M$ sufficiently large, $M \geq \frac{2 \theta}{\eta(\alpha)}$.

Proof of Claim 2. Let $\tilde{y} \in \mathcal{C}_{\alpha}$. There exists $\ell$ affine such that $\eta(\alpha) v(y)+\psi(y) \geq$ $\ell(y)$ for all $y \in T\left(S_{\phi}\left(x_{0}, t_{0}\right)\right)$, and with equality at $\tilde{y}$, and $\ell<-\eta(\alpha)(\theta-1 / 2)$ in $T\left(S_{\phi}\left(x_{0}, t_{0}\right)\right)$. Here $\tilde{y}=T \tilde{x}$ for $\tilde{x} \in S_{\phi}\left(x_{0}, t_{0}\right), S_{\phi}\left(\tilde{x}, \eta_{0}\right) \Subset \Omega$, and we have as in Lemma 4.1 (see proof the of (4.5)) that

$$
\psi(y) \leq \tilde{\ell}_{\tilde{y}}(y)+\frac{1}{t_{0}} d\left(T^{-1} y, T^{-1} \tilde{y}\right)^{2}
$$

for all $y \in T\left(S_{\phi}\left(\tilde{x}, \eta_{0}\right)\right)$ with equality at $\tilde{y}$, and where $\tilde{\ell}_{\tilde{y}}$ is affine given by

$$
\tilde{\ell}_{\tilde{y}}(y)=\psi(\tilde{y})+\nabla \psi(\tilde{y}) \cdot(y-\tilde{y}) .
$$

Therefore

$$
\eta(\alpha) v(y) \geq \ell(y)-\tilde{\ell}_{\tilde{y}}(y)-\frac{1}{t_{0}} d\left(T^{-1} y, T^{-1} \tilde{y}\right)^{2}
$$

for each $y \in T\left(S_{\phi}\left(x_{0}, t_{0}\right)\right)$, and with equality at $y=\tilde{y}$, because $S_{\phi}\left(x_{0}, t_{0}\right) \subset$ $S_{\phi}\left(\tilde{x}, \theta t_{0}\right) \subset S_{\phi}\left(\tilde{x}, \eta_{0}\right)$ for $\theta t_{0}<\eta_{0}$.

Define

$$
g(y)=\ell(y)-\tilde{\ell}_{\tilde{y}}(y)-\frac{1}{t_{0}} d\left(T^{-1} y, T^{-1} \tilde{y}\right)^{2} .
$$

We have from (4.8)

$$
g(\tilde{y})=\eta(\alpha) v(\tilde{y}) \geq \eta(\alpha)\left(\frac{\lambda \theta-\frac{\lambda d\left(T^{-1} \tilde{y}, T^{-1} \bar{y}\right)^{2}}{t_{0}}}{2 \lambda \theta}\right) .
$$

Our goal is to extend the inequality (4.11) to the set $T\left(S_{\phi}\left(\tilde{x}, \theta^{2} /(\lambda M)\right)\right)$ for some $M$ sufficiently large. Note that since $\bar{x}, \tilde{x} \in S_{\phi}\left(x_{0}, t_{0}\right)$ and $t_{0}<1 / \lambda$, by engulfing,

$$
S_{\phi}\left(x_{0}, t_{0}\right) \subset S_{\phi}\left(\bar{x}, \theta t_{0}\right) \subset S_{\phi}(\bar{x}, \theta / \lambda)
$$

and

$$
S_{\phi}\left(x_{0}, t_{0}\right) \subset S_{\phi}\left(\tilde{x}, \theta t_{0}\right) \subset S_{\phi}(\tilde{x}, \theta / \lambda) .
$$

Hence $\bar{x} \in S_{\phi}(\tilde{x}, \theta / \lambda)$, and again by engulfing, $S_{\phi}(\tilde{x}, \theta / \lambda) \subset S_{\phi}\left(\bar{x}, \theta^{2} / \lambda\right)$ and therefore

$$
S_{\phi}\left(\tilde{x}, \theta^{2} /(\lambda M)\right) \subset S_{\phi}(\tilde{x}, \theta / \lambda) \subset S_{\phi}\left(\bar{x}, \theta^{2} / \lambda\right)
$$


for $M \geq \theta$. Therefore to achieve our goal, it is enough to extend the inequality (4.11) to $T\left(S_{\phi}\left(\bar{x}, \theta^{2} / \lambda\right)\right)$; note that $S_{\phi}\left(\bar{x}, \theta^{2} / \lambda\right) \Subset \Omega$.

Let

$$
B=\left\{y \in T\left(S_{\phi}\left(\bar{x}, \theta^{2} / \lambda\right)\right): g(y) \geq \eta(\alpha)\left(\frac{\lambda \theta-\frac{\lambda d\left(T^{-1} y, T^{-1} \bar{y}\right)^{2}}{t_{0}}}{2 \lambda \theta}\right)\right\} .
$$

We claim that

$$
B \subset T\left(S_{\phi}\left(x_{0}, t_{0}\right)\right)
$$

We first prove that

$$
\begin{aligned}
B \subset\{y & \in T\left(S_{\phi}\left(\bar{x}, \theta^{2} / \lambda\right)\right): \\
0 & \left.\geq-\ell(y)+\tilde{\ell}_{\tilde{y}}(y)+\left(\frac{1}{t_{0}}\left(1-\frac{\eta(\alpha)}{2}\right)\right) d\left(T^{-1} y, T^{-1} \tilde{y}\right)^{2}+\frac{\eta(\alpha)}{2}(1-\theta)\right\}
\end{aligned}
$$

$$
=\tilde{B} \text {. }
$$

If $y \in B$, then

$$
0 \geq-\ell(y)+\tilde{\ell}_{\tilde{y}}(y)+\frac{1}{t_{0}} d\left(T^{-1} y, T^{-1} \tilde{y}\right)^{2}-\frac{\eta(\alpha)}{2 \theta} \frac{1}{t_{0}} d\left(T^{-1} y, T^{-1} \bar{y}\right)^{2}+\frac{\eta(\alpha)}{2} .
$$

On the other hand, from Lemma 3.4(a)

$$
\frac{\eta(\alpha)}{2 \theta} \frac{d\left(T^{-1} y, T^{-1} \bar{y}\right)^{2}}{t_{0}} \leq \frac{\eta(\alpha)}{2}\left(\frac{d\left(T^{-1} y, T^{-1} \tilde{y}\right)^{2}}{t_{0}}+\frac{d\left(T^{-1} \bar{y}, T^{-1} \tilde{y}\right)^{2}}{t_{0}}\right) .
$$

Since $d\left(T^{-1} \bar{y}, T^{-1} \tilde{y}\right)^{2} \leq \theta t_{0}$, we get that $y \in \tilde{B}$, and (4.14) follows.

Since $\eta(\alpha) / 2<1$, we have that the function

$$
-\ell(y)+\tilde{\ell}_{\tilde{y}}(y)+\left(\frac{1}{t_{0}}\left(1-\frac{\eta(\alpha)}{2}\right)\right) d\left(T^{-1} y, T^{-1} \tilde{y}\right)^{2}+\frac{\eta(\alpha)}{2}(1-\theta)
$$

is convex and hence $\tilde{B}$ is connected. Note that we have $S_{\phi}\left(\bar{x}, \theta^{2} / \lambda\right) \subset S_{\phi}\left(\tilde{x}, \theta^{3} / \lambda\right)$ $\subset S_{\phi}\left(\tilde{x}, \eta_{0}\right)$ for $\theta^{3} / \lambda \leq \eta_{0}$, and so $d\left(T^{-1} y, T^{-1} \tilde{y}\right)^{2}$ is defined for $y \in T\left(S_{\phi}\left(\bar{x}, \theta^{2} / \lambda\right)\right)$.

Second, we shall prove that

$$
\tilde{B} \subset T\left(S_{\phi}\left(x_{0}, t_{0}\right)\right)
$$

We begin by showing that

$$
\tilde{B} \cap \partial T\left(S_{\phi}\left(x_{0}, t_{0}\right)\right)=\emptyset .
$$

Suppose $y \in \partial T\left(S_{\phi}\left(x_{0}, t_{0}\right)\right)$; then we claim that

$$
-\ell(y)+\tilde{\ell}_{\tilde{y}}(y)+\left(\frac{1}{t_{0}}\left(1-\frac{\eta(\alpha)}{2}\right)\right) d\left(T^{-1} y, T^{-1} \tilde{y}\right)^{2}+\frac{\eta(\alpha)}{2}(1-\theta)>0 .
$$

Recall that $\ell<-\eta(\alpha)(\theta-1 / 2)$ in $\overline{T\left(S_{\phi}\left(x_{0}, t_{0}\right)\right)}$, and $\tilde{\ell}_{\tilde{y}}(y)+\frac{1}{t_{0}} d\left(T^{-1} y, T^{-1} \tilde{y}\right)^{2} \geq$ $\psi(y)=0$. Since $S_{\phi}\left(x_{0}, t_{0}\right) \subset S_{\phi}\left(\tilde{x}, \theta t_{0}\right)$, we have $d\left(T^{-1} y, T^{-1} \tilde{y}\right)^{2} \leq \theta t_{0}$. Hence (4.17) follows, and therefore (4.16) holds. Since $\tilde{y} \in \tilde{B} \cap T\left(S_{\phi}\left(x_{0}, t_{0}\right)\right)$ and $\tilde{B}$ is connected, we get (4.15).

Combining (4.14) with (4.15), we get (4.13) as desired. 
Recall that $\eta(\alpha) v(y) \geq g(y)$ in $T\left(S_{\phi}\left(x_{0}, t_{0}\right)\right)$ by (4.11), and if

$$
y \in T\left(S_{\phi}\left(\bar{x}, \theta^{2} / \lambda\right)\right) \backslash T\left(S_{\phi}\left(x_{0}, t_{0}\right)\right),
$$

then $y \notin B$ by (4.13), that is,

$$
g(y)<\eta(\alpha)\left(\frac{\lambda \theta-\frac{\lambda d\left(T^{-1} y, T^{-1} \bar{y}\right)^{2}}{t_{0}}}{2 \lambda \theta}\right) .
$$

On the other hand, by definition of $G_{\lambda}(u)$ we have from (4.8) that

$$
\tilde{u}(y)-\tilde{u}(\bar{y})-\nabla \tilde{u}(\bar{y}) \cdot(y-\bar{y}) \geq-\lambda \frac{d\left(T^{-1} y, T^{-1} \bar{y}\right)^{2}}{t_{0}}
$$

for all $y \in T\left(S_{\phi}\left(\bar{x}, \theta^{2} / \lambda\right)\right)$, and hence

$$
\frac{\eta(\alpha)}{2 \lambda \theta}\left(\lambda \theta-\frac{d\left(T^{-1} y, T^{-1} \bar{y}\right)^{2}}{t_{0}}\right) \leq \eta(\alpha) v(y),
$$

so $g(y)<\eta(\alpha) v(y)$. Therefore, we have that $g(y) \leq \eta(\alpha) v(y)$ for all $y \in$ $T\left(S_{\phi}\left(\bar{x}, \theta^{2} / \lambda\right)\right)$ with equality at $y=\tilde{y}$. In particular, by (4.12) we have that $g(y) \leq$ $\eta(\alpha) v(y)$ for all $y \in T\left(S_{\phi}\left(\tilde{x}, \theta^{2} /(\lambda M)\right)\right)$ because $S_{\phi}\left(\tilde{x}, \theta^{2} /(\lambda M)\right) \subset S_{\phi}\left(\bar{x}, \theta^{2} / \lambda\right)$ with equality at $\tilde{y}=T \tilde{x}$ for $M \geq \theta$.

Therefore (4.11) holds for all $y \in T\left(S_{\phi}\left(\tilde{x}, \theta^{2} /(\lambda M)\right)\right)$.

Note that from the definitions of $\ell$ and $\tilde{\ell}_{\tilde{y}}$ we have $g(y)=a+p \cdot(y-\tilde{y})-$ $\frac{1}{t_{0}} d\left(T^{-1} y, T^{-1} \tilde{y}\right)^{2}$ with $a=\eta(\alpha) v(\tilde{y})$ and $p=\eta(\alpha) \nabla v(\tilde{y})$. By definition of $v$ we then get that

$$
\tilde{u}(y) \geq \tilde{u}(\tilde{y})+\nabla \tilde{u}(\tilde{y}) \cdot(y-\tilde{y})-\frac{2 \lambda \theta}{t_{0} \eta(\alpha)} d\left(T^{-1} y, T^{-1} \tilde{y}\right)^{2}
$$

for all $y \in T\left(S_{\phi}\left(\tilde{x}, \theta^{2} /(\lambda M)\right)\right.$ for all $M \geq \theta$. Therefore we have proved that

$$
\begin{gathered}
\mathcal{C}_{\alpha} \subset\left\{\tilde{y} \in T\left(S_{\phi}\left(x_{0}, t_{0}\right)\right): \tilde{u}(y) \geq \tilde{u}(\tilde{y})+\nabla \tilde{u}(\tilde{y}) \cdot(y-\tilde{y})-\frac{2 \lambda \theta}{t_{0} \eta(\alpha)} d\left(T^{-1} y, T^{-1} \tilde{y}\right)^{2}\right. \\
\left.\forall y \in T\left(S_{\phi}\left(\tilde{x}, \theta^{2} /(\lambda M)\right)\right) \text { where } \tilde{y}=T \tilde{x}\right\}=F
\end{gathered}
$$

for each $M \geq \theta$. Now

$$
\begin{aligned}
F & =T\left\{\tilde{x} \in S_{\phi}\left(x_{0}, t_{0}\right): u(x) \geq u(\tilde{x})+\nabla u(\tilde{x}) \cdot(x-\tilde{x})-\frac{2 \lambda \theta}{\eta(\alpha)} d(x, \tilde{x})^{2}\right. \\
& \left.\forall x \in S_{\phi}\left(\tilde{x}, \theta^{2} /(\lambda M)\right)\right\} \\
= & T\left(S_{\phi}\left(x_{0}, t_{0}\right) \cap G_{\lambda M}^{-}(u)\right),
\end{aligned}
$$

picking $M=\frac{2 \theta}{\eta(\alpha)}$ (the requirement in the definition of $G$ is satisfied since $S_{\phi}\left(x_{0}, t_{0}\right)$ $\subset \Omega_{\alpha_{0}}$ and $\theta^{2} /(\lambda M)=\eta(\alpha) \theta /(2 \lambda) \leq \eta\left(\alpha_{0}\right)$ since $\lambda$ is sufficiently large). So Claim 2 is proved.

Then from (4.10) and Claim 2 we obtain

$$
\mu\left(S_{\phi}\left(x_{0}, \alpha t_{0}\right)\right) \leq \mu\left(S_{\phi}\left(x_{0}, t_{0}\right) \cap G_{\lambda M}^{-}(u)\right) .
$$

From Lemma 2.5 given $\epsilon>0$ there exists $0<\alpha=\alpha(\epsilon)<1$ such that

$$
(1-\epsilon) \mu\left(S_{\phi}\left(x_{0}, t_{0}\right)\right) \leq \mu\left(S_{\phi}\left(x_{0}, \alpha t_{0}\right)\right) .
$$

The lemma now follows taking $\delta(\epsilon)=\eta(\alpha(\epsilon)) /(2 \theta)$. 


\section{The homogeneous CASE}

Let $u \in C^{2}(\Omega)$ satisfying $L_{\phi} u=0$ in $\Omega$ with $|u| \leq 1$ in $\Omega$. If $0<\alpha_{0}<1$, recall that

$$
\Omega_{\alpha_{0}}=\left\{x \in \Omega: \phi(x)<\left(1-\alpha_{0}\right) \min _{\Omega} \phi\right\} .
$$

Let $0<\epsilon<1 / 2$ and $M$ the largest of the constants in Lemmas 4.1 and 4.2 . Applying Lemma 4.1 to the functions $\frac{u+1}{2}$ and $\frac{-u+1}{2}$, and noting that

$$
G_{M}^{-}((u+1) / 2) \subset G_{2 M}^{-}(u)
$$

and

$$
G_{M}^{-}((-u+1) / 2) \subset G_{2 M}^{+}(u)
$$

we get

$$
\mu\left(S_{\phi}\left(x_{0}, t_{0}\right) \cap G_{2 M / t_{0}}^{-}(u)\right)>(1-\epsilon) \mu\left(S_{\phi}\left(x_{0}, t_{0}\right)\right)
$$

and

$$
\mu\left(S_{\phi}\left(x_{0}, t_{0}\right) \cap G_{2 M / t_{0}}^{+}(u)\right)>(1-\epsilon) \mu\left(S_{\phi}\left(x_{0}, t_{0}\right)\right),
$$

as long as $S_{\phi}\left(x_{0}, t_{0}\right) \subset \Omega_{\alpha_{0}}$ and $t_{0} \theta^{2} / M \leq \eta_{0}$. Now

$$
\begin{aligned}
\mu\left(S_{\phi}\left(x_{0}, t_{0}\right) \backslash G_{2 M / t_{0}}\right) & =\mu\left(S_{\phi}\left(x_{0}, t_{0}\right) \backslash\left(G_{2 M / t_{0}}^{+} \cap G_{2 M / t_{0}}^{-}\right)\right) \\
& =\mu\left(\left(S_{\phi}\left(x_{0}, t_{0}\right) \backslash G_{2 M / t_{0}}^{+}\right) \cup\left(S_{\phi}\left(x_{0}, t_{0}\right) \backslash G_{2 M / t_{0}}^{-}\right)\right) \\
& \leq \mu\left(S_{\phi}\left(x_{0}, t_{0}\right) \backslash G_{2 M / t_{0}}^{+}\right)+\mu\left(S_{\phi}\left(x_{0}, t_{0}\right) \backslash G_{2 M / t_{0}}^{-}\right) \\
& \leq 2 \epsilon \mu\left(S_{\phi}\left(x_{0}, t_{0}\right)\right) .
\end{aligned}
$$

Let $\alpha_{2}<\alpha_{1}<\alpha_{0}$. There exists $\eta_{2}$ such that if $x \in \Omega_{\alpha_{2}}$ and $t \leq \eta_{2}$, then $S_{\phi}(x, t) \subset$ $\Omega_{\alpha_{1}}$, and there exists $\eta_{1}$ such that if $x \in \Omega_{\alpha_{1}}$ and $t \leq \eta_{1}$, then $S_{\phi}(x, t) \subset \Omega_{\alpha_{0}}$.

Let $\theta^{3} / \lambda \leq \eta_{2} / 2$ and let $x_{0} \in \Omega_{\alpha_{2}} \backslash G_{\lambda M}(u)$.

Define

$$
g(t)=\frac{\mu\left(\left(\Omega_{\alpha_{2}} \backslash G_{\lambda M}(u)\right) \cap S_{\phi}\left(x_{0}, t\right)\right)}{\mu\left(S_{\phi}\left(x_{0}, t\right)\right)} .
$$

We have $\lim _{t \rightarrow 0} g(t)=1$. Also, if $2 / \lambda \leq t<\eta_{1}$, then $S_{\phi}\left(x_{0}, t\right) \subset \Omega_{\alpha_{0}}$ and

$$
\begin{aligned}
\mu\left(\left(\Omega_{\alpha_{2}} \backslash G_{\lambda M}(u)\right) \cap S_{\phi}\left(x_{0}, t\right)\right) & \leq \mu\left(S_{\phi}\left(x_{0}, t\right) \backslash G_{\lambda M}(u)\right) \\
& \leq \mu\left(S_{\phi}\left(x_{0}, t\right) \backslash G_{2 M / t}(u)\right) \\
& \leq 2 \epsilon \mu\left(S_{\phi}\left(x_{0}, t\right)\right),
\end{aligned}
$$

since $G_{2 M / t}(u) \subset G_{\lambda M}(u)$. Therefore $g(t) \leq 2 \epsilon$ for $t \in\left[2 / \lambda, \eta_{1}\right)$. Then by continuity of $g$, there exists $t_{x_{0}} \leq 2 / \lambda \leq \theta^{3} / \lambda \leq \eta_{2} / 2$ such that $g\left(t_{x_{0}}\right)=2 \epsilon$.

Then we have proved that if $0<\epsilon<1 / 2$ and $M$ is the constant in Lemma 4.1, then for $\alpha_{2}<\alpha_{1}<\alpha_{0}$ and for any $\lambda>0$ such that $\theta^{3} / \lambda \leq \eta_{2} / 2$ and for any $x_{0} \in \Omega_{\alpha_{2}} \backslash G_{\lambda M}(u)$ there exists $t_{x_{0}} \leq 2 / \lambda$ such that

$$
\mu\left(\left(\Omega_{\alpha_{2}} \backslash G_{\lambda M}(u)\right) \cap S_{\phi}\left(x_{0}, t_{x_{0}}\right)\right)=2 \epsilon \mu\left(S_{\phi}\left(x_{0}, t_{x_{0}}\right)\right) .
$$

We now claim that (5.1) implies that

$$
S_{\phi}\left(x_{0}, t_{x_{0}}\right) \subset \Omega_{\alpha_{1}} \backslash G_{\lambda}(u) .
$$

Suppose that (5.2) is false. Since $x_{0} \in \Omega_{\alpha_{2}}$ and $t_{x_{0}} \leq 2 / \lambda \leq \eta_{2}$, we have that $S_{\phi}\left(x_{0}, t_{x_{0}}\right) \subset \Omega_{\alpha_{1}}$. Therefore, if $S_{\phi}\left(x_{0}, t_{x_{0}}\right) \nsubseteq \Omega_{\alpha_{1}} \backslash G_{\lambda}(u)$, then $S_{\phi}\left(x_{0}, t_{x_{0}}\right) \cap$ 
$G_{\lambda}(u) \neq \emptyset$ and hence from Lemma 4.2 applied to $u$ and $-u$, we have for $M$ sufficiently large,

$$
(1-\epsilon) \mu\left(S_{\phi}\left(x_{0}, t_{x_{0}}\right)\right)<\mu\left(S_{\phi}\left(x_{0}, t_{x_{0}}\right) \cap G_{\lambda M}^{-}(u)\right)
$$

and

$$
(1-\epsilon) \mu\left(S_{\phi}\left(x_{0}, t_{x_{0}}\right)\right)<\mu\left(S_{\phi}\left(x_{0}, t_{x_{0}}\right) \cap G_{\lambda M}^{+}(u)\right) ;
$$

note that $G_{\lambda M}^{-}(-u)=G_{\lambda M}^{+}(u)$ and $G_{\lambda}(-u)=G_{\lambda}(u)$. Therefore

$$
\mu\left(S_{\phi}\left(x_{0}, t_{x_{0}}\right) \backslash G_{\lambda M}(u)\right)<2 \epsilon \mu\left(S_{\phi}\left(x_{0}, t_{x_{0}}\right),\right.
$$

and so

$$
\mu\left(\left(\Omega_{\alpha_{2}} \backslash G_{\lambda M}(u)\right) \cap S_{\phi}\left(x_{0}, t_{x_{0}}\right)\right) \leq \mu\left(S_{\phi}\left(x_{0}, t_{x_{0}}\right) \backslash G_{\lambda M}(u)\right)<2 \epsilon \mu\left(S_{\phi}\left(x_{0}, t_{x_{0}}\right),\right.
$$

contradicting (5.1). This proves (5.2).

Then from Lemma 2.7 we get that

$$
\mu\left(\Omega_{\alpha_{2}} \backslash G_{\lambda M}(u)\right) \leq \delta(\epsilon) \mu\left(\Omega_{\alpha_{1}} \backslash G_{\lambda}(u)\right),
$$

where $\alpha_{2}<\alpha_{1}<\alpha_{0}, \theta^{3} / \lambda \leq \eta_{2} / 2$, and $M=M(\epsilon)$ large, for all $\epsilon$ sufficiently small.

5.1. Power decay. Recalling Lemma 2.3, we can apply (5.3) with $\lambda=M$ and

$$
\frac{\theta^{3}}{M}=\frac{C_{0}\left(\alpha_{1}-\alpha_{2}\right)^{\gamma}}{2}=\frac{\eta_{2}}{2}
$$

that is,

$$
\alpha_{2}=\alpha_{1}-\left(\frac{2 \theta^{3}}{C_{0} M}\right)^{1 / \gamma}
$$

Then

$$
\mu\left(\Omega_{\alpha_{2}} \backslash G_{M^{2}}(u)\right) \leq \delta(\epsilon) \mu\left(\Omega_{\alpha_{1}} \backslash G_{M}(u)\right) .
$$

Next apply (5.3) with $\lambda=M^{2}$ and $\frac{\theta^{3}}{M}=\frac{C_{0}\left(\alpha_{2}-\alpha_{3}\right)^{\gamma}}{2}=\frac{\eta_{3}}{2}$, that is,

$$
\alpha_{3}=\alpha_{2}-\left(\frac{2 \theta^{3}}{C_{0} M^{2}}\right)^{1 / \gamma}=\alpha_{1}-\left(\frac{2 \theta^{3}}{C_{0} M}\right)^{1 / \gamma}-\left(\frac{2 \theta^{3}}{C_{0} M^{2}}\right)^{1 / \gamma} \text {. }
$$

Then

$$
\mu\left(\Omega_{\alpha_{3}} \backslash G_{M^{3}}(u)\right) \leq \delta(\epsilon) \mu\left(\Omega_{\alpha_{2}} \backslash G_{M^{2}}(u)\right) \leq(\delta(\epsilon))^{2} \mu\left(\Omega_{\alpha_{1}} \backslash G_{M}(u)\right) .
$$

Continuing in this way, if

$$
\alpha_{k}=\alpha_{1}-\sum_{j=1}^{k-1}\left(\frac{2 \theta^{3}}{C_{0} M^{j}}\right)^{1 / \gamma}
$$

then

$$
\mu\left(\Omega_{\alpha_{k}} \backslash G_{M^{k}}(u)\right) \leq(\delta(\epsilon))^{k-1} \mu\left(\Omega_{\alpha_{1}} \backslash G_{M}(u)\right) .
$$

Now $\sum_{j=1}^{\infty}\left(\frac{1}{M^{1 / \gamma}}\right)^{j}=\frac{1}{M^{1 / \gamma}-1}$, and so

$$
\mu\left(\Omega_{\alpha_{1}-\frac{C}{M^{1 / \gamma}-1}} \backslash G_{M^{k}}(u)\right) \leq(\delta(\epsilon))^{k-1} \mu\left(\Omega_{\alpha_{1}} \backslash G_{M}(u)\right)
$$

for $k=1,2, \cdots$. 
We then obtain the following theorem.

Theorem 5.1. Let $\Omega$ be a normalized convex domain, $\phi \in C^{2}(\Omega)$ convex, $\phi=0$ on $\partial \Omega, \lambda \leq \operatorname{det} D^{2} \phi \leq \Lambda, u \in C^{2}(\Omega),|u| \leq 1$ in $\Omega$, a solution to $L_{\phi} u=0$ in $\Omega$. Then for each $\epsilon>0$ and $0<\alpha_{0}<1$ there exists a constant $M=M\left(\epsilon, \alpha_{0}, \lambda, \Lambda\right)$ such that for all $u$ and $\phi$ as above we have

$$
\mu\left(\Omega_{\alpha_{0} / 2} \backslash G_{M^{k}}(u)\right) \leq(\delta(\epsilon))^{k-1} \mu\left(\Omega_{\alpha_{0}} \backslash G_{M}(u)\right)
$$

for $k=1,2, \cdots$.

This statement implies the following. Let $t \geq M$ and pick $k$ such that $M^{k} \leq t \leq$ $M^{k+1}$. Then $G_{M^{k}}(u) \subset G_{t}(u) \subset G_{M^{k+1}}(u)$ and $k \leq \log _{M} t \leq k+1$. So

$$
\begin{aligned}
\mu\left(\Omega_{\alpha_{0} / 2} \backslash G_{t}(u)\right) & \leq \mu\left(\Omega_{\alpha_{0} / 2} \backslash G_{M^{k}}(u)\right) \\
& \leq(\delta(\epsilon))^{k-1} \mu\left(\Omega_{\alpha_{0}} \backslash G_{M}(u)\right) \\
& \leq(\delta(\epsilon))^{k-1} \mu\left(\Omega_{\alpha_{0}}\right)=\frac{1}{\delta(\epsilon)^{2}}(\delta(\epsilon))^{k+1} \mu\left(\Omega_{\alpha_{0}}\right) \\
& \leq \frac{1}{\delta(\epsilon)^{2}}(\delta(\epsilon))^{\log _{M} t} \mu\left(\Omega_{\alpha_{0}}\right)=\frac{1}{\delta(\epsilon)^{2}} t \frac{\ln \delta(\epsilon)}{\ln M} \mu\left(\Omega_{\alpha_{0}}\right) .
\end{aligned}
$$

Thus, combining this estimate with Remark 3.8 and the inclusion (3.5), we obtain our main theorem in the homogeneous case.

Theorem 5.2. Let $\Omega$ be a normalized convex domain, $\phi \in C^{2}(\Omega), \phi=0$ on $\partial \Omega$, and $\lambda \leq \operatorname{det} D^{2} \phi \leq \Lambda$. Suppose $u \in C^{2}(\Omega)$, and $L_{\Phi} u=0$ in $\Omega$. Then, given $0<\alpha_{0}<1$ there exist positive constants $p$ and $C$ depending only on $\lambda, \Lambda$, and $\alpha_{0}$ such that

$$
\left\|D^{2} u\right\|_{L^{p}\left(\Omega_{\alpha_{0} / 2}\right)} \leq C\|u\|_{L^{\infty}(\Omega)}
$$

Proof. Let $v=\frac{u}{\|u\|_{L^{\infty}(\Omega)}}$. We can apply the above estimate to $v$ to get

$$
\left\|D^{2} v\right\|_{L^{p}\left(\Omega_{\alpha_{0} / 2}, d \mu\right)} \leq C
$$

for some $p$ and $C$ depending on $\lambda, \Lambda$, and $\alpha_{0}$, which implies the theorem, since in our case Lebesgue measure is comparable to the Monge-Ampère measure $\mu$.

\section{The nonhomogeneous CASE}

Lemma 6.1. Let $\Omega$ be a normalized convex domain, $\phi \in C^{2}(\Omega), \phi=0$ on $\partial \Omega$, and $\lambda \leq \operatorname{det} D^{2} \phi \leq \Lambda$. Suppose $u \in C^{2}(\Omega), 0 \leq u \leq 1$, and $L_{\phi} u=f$ in $\Omega$. Assume that $\left\|f / \operatorname{det} D^{2} \phi\right\|_{L^{n}(\Omega)} \leq 1$

Then for each $\epsilon>0$ there exist positive constants $\eta(\epsilon, \lambda, \Lambda)$ and $C(\lambda, \Lambda, n)$ such that for each $\eta \leq \eta(\epsilon, \lambda, \Lambda)$ and for each section $S_{\phi}\left(x_{0}, t_{0}\right) \subset \Omega_{\alpha_{0}}$, we have

$$
\text { (6.1) } \mu\left(G_{1 /\left(\eta t_{0}\right)}^{-}(u) \cap S_{\phi}\left(x_{0}, t_{0}\right)\right)>\left((1-\epsilon)^{1 / n}-\eta t_{0}^{1 / 2} C(\lambda, \Lambda, n)\right)^{n} \mu\left(S_{\phi}\left(x_{0}, t_{0}\right)\right) \text {. }
$$

Proof. We use the notation from the proof of Lemma 4.1. The proof proceeds in the same way, in particular, Claims 1 and 2 hold; the only difference is to handle the integral $\int_{\tilde{\Omega}_{\alpha}} \operatorname{det} D^{2} \tilde{\phi}(y) d y$.

Let $\tilde{\Phi}(y)=\operatorname{det} D^{2} \tilde{\phi}(y)\left(D^{2} \tilde{\phi}(y)\right)^{-1}$. We have

$$
D^{2} \tilde{\phi}(y)=\frac{1}{t_{0}}\left(T^{-1}\right)^{t} D^{2} \phi\left(T^{-1} y\right) T^{-1}
$$


and $D^{2} \tilde{u}(y)=\left(T^{-1}\right)^{t} D^{2} u\left(T^{-1} y\right) T^{-1}$. So

$$
\operatorname{det} D^{2} \tilde{\phi}(y)=\frac{1}{t_{0}^{n}|\operatorname{det} T|^{2}} \operatorname{det} D^{2} \phi\left(T^{-1} y\right)
$$

and $\left(D^{2} \tilde{\phi}(y)\right)^{-1}=t_{0} T\left(D^{2} \phi\left(T^{-1} y\right)\right)^{-1} T^{t}$. Hence

$$
\operatorname{trace}\left(\tilde{\Phi}(y) D^{2} \tilde{u}(y)\right)=\frac{1}{t_{0}^{n-1}|\operatorname{det} T|^{2}} f\left(T^{-1} y\right)
$$

and

$$
\operatorname{det} \tilde{\Phi}(y)=\left(\frac{1}{t_{0}^{n}|\operatorname{det} T|^{2}}\right)^{n-1}\left(\operatorname{det} D^{2} \phi\left(T^{-1} y\right)\right)^{n-1} .
$$

Applying Lemma 3.1 with $u \rightsquigarrow \eta(\alpha) \tilde{u}, \phi \rightsquigarrow \tilde{\psi}, E=\mathcal{C}_{\alpha}, F=\tilde{\Omega}_{\alpha}$, and using Minkowski's inequality we get

$$
\begin{gathered}
\left(\int_{\tilde{\Omega}_{\alpha}} \operatorname{det} D^{2} \tilde{\phi}(y) d y\right)^{1 / n} \leq \\
+\frac{\eta(\alpha)}{n} \frac{1}{|\operatorname{det} T|^{2 / n}}\left(\int_{\mathcal{C}_{\alpha}} \frac{\left|f\left(T^{-1} y\right)\right|^{n}}{\left(\operatorname{det} D^{2} \phi\left(T^{-1} y\right)\right)^{n-1}} d y\right)^{1 / n} \\
+\left(\int_{\mathcal{C}_{\alpha}} \operatorname{det} D^{2} \tilde{\phi}(y) d y\right)^{1 / n} .
\end{gathered}
$$

Since by Claim 2, $\mathcal{C}_{\alpha} \subset T\left(S_{\phi}\left(x_{0}, t_{0}\right) \cap G_{1 /\left(\eta(\alpha) t_{0}\right)}^{-}(u)\right)$, we get

$$
\begin{aligned}
& \left(\int_{T\left(S_{\phi}\left(x_{0}, \alpha t_{0}\right)\right)} \operatorname{det} D^{2} \tilde{\phi}(y) d y\right)^{1 / n} \\
& \leq \frac{\eta(\alpha)}{n} \frac{1}{|\operatorname{det} T|^{2 / n}}\left(\int_{T\left(S_{\phi}\left(x_{0}, t_{0}\right) \cap G_{1 /\left(\eta(\alpha) t_{0}\right)}^{-}(u)\right)} \frac{\left|f\left(T^{-1} y\right)\right|^{n}}{\left(\operatorname{det} D^{2} \phi\left(T^{-1} y\right)\right)^{n-1}} d y\right)^{1 / n} \\
& \quad+\left(\int_{T\left(S_{\phi}\left(x_{0}, t_{0}\right) \cap G_{1 /\left(\eta(\alpha) t_{0}\right)}^{-}(u)\right)} \operatorname{det} D^{2} \tilde{\phi}(y) d y\right)^{1 / n} .
\end{aligned}
$$

Changing variables yields

$$
\begin{aligned}
& \left(\int_{S_{\phi}\left(x_{0}, \alpha t_{0}\right)} \operatorname{det} D^{2} \phi(x) d x\right)^{1 / n} \\
& \leq \frac{\eta(\alpha)}{n} t_{0}\left(\int_{S_{\phi}\left(x_{0}, t_{0}\right) \cap G_{1 /\left(\eta(\alpha) t_{0}\right)}^{-}(u)} \frac{|f(x)|^{n}}{\left.\operatorname{det} D^{2} \phi(x)\right)^{n-1}} d x\right)^{1 / n} \\
& \quad+\left(\int_{S_{\phi}\left(x_{0}, t_{0}\right) \cap G_{1 /\left(\eta(\alpha) t_{0}\right)}^{-}(u)} \operatorname{det} D^{2} \phi(x) d x\right)^{1 / n}
\end{aligned}
$$

That is,

$$
\begin{aligned}
& \mu\left(S_{\phi}\left(x_{0}, \alpha t_{0}\right)\right)^{1 / n} \\
& \leq \frac{\eta(\alpha)}{n} t_{0}\left\|f /\left(\operatorname{det} D^{2} \phi\right)\right\|_{L^{n}(\Omega)}+\mu\left(S_{\phi}\left(x_{0}, t_{0}\right) \cap G_{1 /\left(\eta(\alpha) t_{0}\right)}^{-}(u)\right)^{1 / n} \\
& \leq \frac{\eta(\alpha)}{n} t_{0}+\mu\left(S_{\phi}\left(x_{0}, t_{0}\right) \cap G_{1 /\left(\eta(\alpha) t_{0}\right)}^{-}(u)\right)^{1 / n} \\
& \leq \frac{\eta(\alpha)}{n} t_{0}^{1 / 2} C(n, \lambda, \Lambda) \mu\left(S_{\phi}\left(x_{0}, t_{0}\right)\right)^{1 / n}+\mu\left(S_{\phi}\left(x_{0}, t_{0}\right) \cap G_{1 /\left(\eta(\alpha) t_{0}\right)}^{-}(u)\right)^{1 / n} .
\end{aligned}
$$


Note that this inequality holds for any $\eta \leq \eta(\alpha)$. Given $0<\epsilon<1 / 2$ pick $\alpha=\alpha(\epsilon)$ such that

$$
(1-\epsilon) \mu\left(S_{\phi}\left(x_{0}, t_{0}\right)\right) \leq \mu\left(S_{\phi}\left(x_{0}, \alpha t_{0}\right)\right)
$$

which combined with the previous inequality yields the lemma for any $\eta \leq \eta(\alpha(\epsilon))$.

Lemma 6.2. Let $\Omega$ be a normalized convex domain, $\phi \in C^{2}(\Omega), \phi=0$ on $\partial \Omega$, and $\lambda \leq \operatorname{det} D^{2} \phi \leq \Lambda$. Suppose $u \in C^{2}(\Omega)$, and $L_{\phi} u=f$ in $\Omega$.

Then for each $0<\epsilon<1 / 2$ there exist positive constants $\eta(\epsilon, \lambda, \Lambda)$ and $C(\epsilon, \lambda, \Lambda)$ such that if $S_{\phi}\left(x_{0}, t_{0}\right) \subset \Omega_{\alpha_{0}}, t_{0}<1 / \lambda<\eta_{0} / \theta^{3}, \bar{x} \in S_{\phi}\left(x_{0}, t_{0}\right) \cap G_{\lambda}(u)$, and $\mathcal{M}_{\mu}\left(\left(f / \operatorname{det} D^{2} \phi\right)^{n}\right)(\bar{x}) \leq(2 \theta n \lambda)^{n}$, then

$$
\left.\mu\left(G_{2 \lambda \theta / \eta}^{-}(u) \cap S_{\phi}\left(x_{0}, t_{0}\right)\right)>\left((1-\epsilon)^{1 / n}-C(\epsilon, \lambda, \Lambda) \eta\right)\right)^{n} \mu\left(S_{\phi}\left(x_{0}, t_{0}\right)\right.
$$

for $\eta \leq \eta(\epsilon, \lambda, \Lambda)$.

Proof. We use the notation from Lemma 4.2 and note that Claims 1 and 2 hold. As in the proof of (6.2) we get

$$
\begin{aligned}
\mu & \left(S_{\phi}\left(x_{0}, \alpha t_{0}\right)\right)^{1 / n} \\
\leq & \frac{\eta(\alpha)}{n} \frac{1}{2 \lambda \theta}\left(\int_{S_{\phi}\left(x_{0}, t_{0}\right)} \frac{f(x)^{n}}{\left(\operatorname{det} D^{2} \phi(x)\right)^{n-1}} d x\right)^{1 / n} \\
& \quad+\mu\left(S_{\phi}\left(x_{0}, t_{0}\right) \cap G_{2 \lambda \theta / \eta(\alpha)}^{-}(u)\right)^{1 / n} \\
\leq & \frac{\eta(\alpha)}{n} \frac{1}{2 \lambda \theta} \mu\left(S_{\phi}\left(\bar{x}, \theta t_{0}\right)\right)^{1 / n} \\
\quad & \left(\frac{1}{\mu\left(S_{\phi}\left(\bar{x}, \theta t_{0}\right)\right)} \int_{S_{\phi}\left(\bar{x}, \theta t_{0}\right)} \operatorname{det} D^{2} \phi(x) \frac{f(x)^{n}}{\left(\operatorname{det} D^{2} \phi(x)\right)^{n}} d x\right)^{1 / n} \\
& \quad+\mu\left(S_{\phi}\left(x_{0}, t_{0}\right) \cap G_{2 \lambda \theta / \eta(\alpha)}^{-}(u)\right)^{1 / n} \\
\leq & \frac{\eta(\alpha)}{n} \frac{1}{2 \lambda \theta} \mu\left(S_{\phi}\left(\bar{x}, \theta t_{0}\right)\right)^{1 / n} \mathcal{M}_{\mu}\left(\left(f / \operatorname{det} D^{2} \phi\right)^{n}\right)(\bar{x})^{1 / n} \\
& +\mu\left(S_{\phi}\left(x_{0}, t_{0}\right) \cap G_{2 \lambda \theta / \eta(\alpha)}^{-}(u)\right)^{1 / n} \\
\leq & C(\lambda, \Lambda) \eta(\alpha) \mu\left(S_{\phi}\left(x_{0}, t_{0}\right)\right)^{1 / n}+\mu\left(S_{\phi}\left(x_{0}, t_{0}\right) \cap G_{2 \lambda \theta / \eta(\alpha)}^{-}(u)\right)^{1 / n} .
\end{aligned}
$$

Note that in the above inequality, $\eta(\alpha)$ can be replaced by any $\eta \leq \eta(\alpha)$. Therefore picking $\alpha=\alpha(\epsilon)$ as in the proof of the previous lemma we get

$$
\mu\left(S_{\phi}\left(x_{0}, t_{0}\right)\right)\left((1-\epsilon)^{1 / n}-C(n, \lambda, \Lambda) \eta\right)^{n} \leq \mu\left(S_{\phi}\left(x_{0}, t_{0}\right) \cap G_{2 \lambda \theta / \eta}^{-}(u)\right)
$$

for each $\eta \leq \eta(\alpha)$.

6.1. Power decay for the nonhomogeneous case. Let $\Omega$ be a normalized convex domain, $\phi \in C^{2}(\Omega)$ convex, $\phi=0$ on $\partial \Omega, \lambda \leq \operatorname{det} D^{2} \phi \leq \Lambda, u \in C^{2}(\Omega),|u| \leq 1$ in $\Omega, L_{\phi} u=f$ in $\Omega$ and suppose that $\left\|f / \operatorname{det} D^{2} \phi\right\|_{L^{n}(\Omega, \mu)} \leq 1$. Given $0<\epsilon<1 / 4$, we pick $\eta$ sufficiently small depending on $\epsilon$ such that from Lemma 6.1 we get with $M=1 / \eta$ and for $S_{\phi}\left(x_{0}, t_{0}\right) \subset \Omega_{\alpha_{0}}$ that

$$
\frac{3}{4}(1-\epsilon) \mu\left(S_{\phi}\left(x_{0}, t_{0}\right)\right) \leq \mu\left(S_{\phi}\left(x_{0}, t_{0}\right) \cap G_{M / t_{0}}^{-}(u)\right)
$$


and

$$
\frac{3}{4}(1-\epsilon) \mu\left(S_{\phi}\left(x_{0}, t_{0}\right)\right) \leq \mu\left(S_{\phi}\left(x_{0}, t_{0}\right) \cap G_{M / t_{0}}^{+}(u)\right) .
$$

Hence

$$
\begin{aligned}
\mu\left(S_{\phi}\left(x_{0}, t_{0}\right) \backslash G_{M / t_{0}}(u)\right) & =\mu\left(S_{\phi}\left(x_{0}, t_{0}\right) \backslash\left(G_{M / t_{0}}^{+}(u) \cap G_{M / t_{0}}^{-}(u)\right)\right) \\
& \leq \mu\left(S_{\phi}\left(x_{0}, t_{0}\right) \backslash G_{M / t_{0}}^{+}(u)\right)+\mu\left(S_{\phi}\left(x_{0}, t_{0}\right) \backslash G_{M / t_{0}}^{-}(u)\right) \\
& \leq\left(2-\frac{3}{2}(1-\epsilon)\right) \mu\left(S_{\phi}\left(x_{0}, t_{0}\right)\right)=\delta(\epsilon) \mu\left(S_{\phi}\left(x_{0}, t_{0}\right)\right) .
\end{aligned}
$$

Proceeding as in Section 5 we get that given $0<\epsilon<1 / 4$ there exists $M=$ $M(\epsilon, \lambda, \Lambda)$ as in Lemmas 6.1 and 6.2 such that for $\alpha_{2}<\alpha_{1}<\alpha_{0}$ and for $\theta^{3} / \lambda \leq \eta_{2} / 2$ and for any $x_{0} \in \Omega_{\alpha_{2}} \backslash G_{\lambda M}(u)$ there exists $t_{x_{0}} \leq 1 / \lambda$ such that

$$
\mu\left(\left(\Omega_{\alpha_{2}} \backslash G_{\lambda M}(u)\right) \cap S_{\phi}\left(x_{0}, t_{x_{0}}\right)\right)=\delta(\epsilon) \mu\left(S_{\phi}\left(x_{0}, t_{x_{0}}\right)\right) .
$$

We now claim that if $x_{0} \in \Omega_{\alpha_{2}} \backslash G_{\lambda M}(u), \theta^{3} / \lambda \leq \eta_{2} / 2$ and (6.4) holds, then

$$
S_{\phi}\left(x_{0}, t_{x_{0}}\right) \subset\left(\Omega_{\alpha_{1}} \backslash G_{\lambda}(u)\right) \cup\left\{x \in \Omega_{\alpha_{0}}: \mathcal{M}_{\mu}\left(\left(f / \operatorname{det} D^{2} \phi\right)^{n}\right)(x)>(2 \theta n \lambda)^{n}\right\} .
$$

Otherwise, and since $x_{0} \in \Omega_{\alpha_{2}}$ and $t_{x_{0}} \leq 1 / \lambda<\eta_{2}$, we have that $S_{\phi}\left(x_{0}, t_{x_{0}}\right) \subset$ $\Omega_{\alpha_{1}}$ and there exists $\bar{x} \in S_{\phi}\left(x_{0}, t_{x_{0}}\right) \cap G_{\lambda}(u)$ such that $\mathcal{M}_{\mu}\left(\left(f / \operatorname{det} D^{2} \phi\right)^{n}\right)(\bar{x}) \leq$ $(2 \theta n \lambda)^{n}$. Then by Lemma 6.2 we get that

$$
\frac{3}{4}(1-\epsilon) \mu\left(S_{\phi}\left(x_{0}, t_{x_{0}}\right)\right)<\mu\left(S_{\phi}\left(x_{0}, t_{x_{0}}\right) \cap G_{\lambda M}^{-}(u)\right)
$$

and

$$
\frac{3}{4}(1-\epsilon) \mu\left(S_{\phi}\left(x_{0}, t_{x_{0}}\right)\right)<\mu\left(S_{\phi}\left(x_{0}, t_{x_{0}}\right) \cap G_{\lambda M}^{+}(u)\right) .
$$

Hence

$$
\mu\left(\left(\Omega_{\alpha_{2}} \backslash G_{\lambda M}(u)\right) \cap S_{\phi}\left(x_{0}, t_{x_{0}}\right)\right) \leq \mu\left(S_{\phi}\left(x_{0}, t_{x_{0}}\right) \backslash G_{\lambda M}(u)\right)<\delta(\epsilon) \mu\left(S_{\phi}\left(x_{0}, t_{x_{0}}\right)\right),
$$

a contradiction with (6.4). So (6.5) is proved.

Hence by Lemma 2.7 there exist $0<\tilde{\delta}(\epsilon)<1$ and a family of sections $S_{\phi}\left(x_{k}, t_{k}\right)$ whose union covers $\Omega_{\alpha_{2}} \backslash G_{\lambda M}(u)$ satisfying (6.4) and

$$
\begin{aligned}
& \mu\left(\Omega_{\left.\alpha_{2} \backslash G_{\lambda M}(u)\right) \leq} \leq \tilde{\delta}(\epsilon) \mu\left(\bigcup_{k=1}^{\infty} S_{\phi}\left(x_{k}, t_{k}\right)\right)\right. \\
& \leq \tilde{\delta}(\epsilon) \mu\left(\Omega_{\alpha_{1}} \backslash G_{\lambda}(u)\right) \\
& \quad+\tilde{\delta}(\epsilon) \mu\left\{x \in \Omega_{\alpha_{0}}: \mathcal{M}_{\mu}\left(\left(f / \operatorname{det} D^{2} \phi\right)^{n}\right)(x)>(2 \theta n \lambda)^{n}\right\},
\end{aligned}
$$

by (6.5).

We now proceed as in Subsection [5.1. Fix $\alpha_{1}<\alpha_{0}$ and let $\lambda=M$, and set $\alpha_{2}=\alpha_{1}-\left(\frac{\theta^{3}}{C_{0} M}\right)^{1 / \gamma}$, where $\gamma$ and $C_{0}$ are the constants in Lemma 2.1. Then $\frac{\theta^{3}}{\lambda}=\frac{\theta^{3}}{M}=C_{0}\left(\alpha_{1}-\alpha_{0}\right)^{\gamma}=\eta_{2}$, and so from (6.6) we get

$$
\begin{aligned}
\mu\left(\Omega_{\alpha_{2}} \backslash G_{M^{2}}(u)\right) \leq \tilde{\delta} & (\epsilon) \mu\left(\Omega_{\alpha_{1}} \backslash G_{M}(u)\right) \\
& +\tilde{\delta}(\epsilon) \mu\left\{x \in \Omega_{\alpha_{0}}: \mathcal{M}_{\mu}\left(\left(f / \operatorname{det} D^{2} \phi\right)^{n}\right)(x)>(2 \theta n M)^{n}\right\} .
\end{aligned}
$$


Next let $\lambda=M^{2}$ and $\alpha_{3}=\alpha_{2}-\left(\frac{\theta^{3}}{C_{0} M^{2}}\right)^{1 / \gamma}$, so $\frac{\theta^{3}}{\lambda}=\frac{\theta^{3}}{M^{2}}=C_{0}\left(\alpha_{2}-\alpha_{3}\right)^{\gamma}=\eta_{3}$. Then

$$
\begin{aligned}
\mu\left(\Omega_{\alpha_{3}} \backslash G_{M^{3}}(u)\right) \leq \tilde{\delta} & (\epsilon) \mu\left(\Omega_{\alpha_{2}} \backslash G_{M^{2}}(u)\right) \\
& +\tilde{\delta}(\epsilon) \mu\left\{x \in \Omega_{\alpha_{0}}: \mathcal{M}_{\mu}\left(\left(f / \operatorname{det} D^{2} \phi\right)^{n}\right)(x)>\left(2 \theta n M^{2}\right)^{n}\right\} \\
\leq & \tilde{\delta}(\epsilon)^{2} \mu\left(\Omega_{\alpha_{1}} \backslash G_{M}(u)\right) \\
& +\tilde{\delta}(\epsilon)^{2} \mu\left\{x \in \Omega_{\alpha_{0}}: \mathcal{M}_{\mu}\left(\left(f / \operatorname{det} D^{2} \phi\right)^{n}\right)(x)>(2 \theta n M)^{n}\right\} \\
& +\tilde{\delta}(\epsilon) \mu\left\{x \in \Omega_{\alpha_{0}}: \mathcal{M}_{\mu}\left(\left(f / \operatorname{det} D^{2} \phi\right)^{n}\right)(x)>\left(2 \theta n M^{2}\right)^{n}\right\}
\end{aligned}
$$

Continuing in this way we let $\lambda=M^{k}$ and $\alpha_{k+1}=\alpha_{k}-\left(\frac{\theta^{3}}{C_{0} M^{k}}\right)^{1 / \gamma}$. Then $\frac{\theta^{3}}{\lambda}=$ $\frac{\theta^{3}}{M^{k}}=C_{0}\left(\alpha_{k}-\alpha_{k+1}\right)^{\gamma}=\eta_{k}$, and

$$
\begin{aligned}
& \mu\left(\Omega_{\alpha_{k+1}} \backslash G_{M^{k+1}}(u)\right) \\
& \leq \tilde{\delta}^{k} \mu\left(\Omega_{\alpha_{1}} \backslash G_{M}(u)\right) \\
& \quad+\sum_{j=1}^{k} \tilde{\delta}^{j} \mu\left\{x \in \Omega_{\alpha_{0}}: \mathcal{M}_{\mu}\left(\left(f / \operatorname{det} D^{2} \phi\right)^{n}\right)(x)>C\left(M^{n}\right)^{k+1-j}\right\} .
\end{aligned}
$$

Since $\mathcal{M}_{\mu}$ is of weak type $1-1$ by Theorem 2.9 , we get

$$
\begin{aligned}
& \mu\left\{x \in \Omega_{\alpha_{0}}: \mathcal{M}_{\mu}\left(\left(f / \operatorname{det} D^{2} \phi\right)^{n}\right)(x)>C\left(M^{n}\right)^{k+1-j}\right\} \\
& \leq \frac{C_{n}}{\left(M^{n}\right)^{k+1-j}} \int_{\Omega}\left|\frac{f}{\operatorname{det} D^{2} \phi}\right|^{n} d \mu(x) \leq \frac{C_{n}}{\left(M^{n}\right)^{k+1-j}} .
\end{aligned}
$$

Set $m_{0}=\max \left\{\tilde{\delta}, M^{-n}\right\}$. Then we have

$$
\begin{aligned}
& \sum_{j=1}^{k} \tilde{\delta}^{j} \mu\left\{x \in \Omega_{\alpha_{0}}: \mathcal{M}_{\mu}\left(\left(f / \operatorname{det} D^{2} \phi\right)^{n}\right)(x)>C\left(M^{n}\right)^{k+1-j}\right\} \\
& \quad \leq C_{n} \sum_{j=1}^{k} m_{0}^{k+1}=C_{n} k m_{0}^{k+1} .
\end{aligned}
$$

Hence

$$
\mu\left(\Omega_{\alpha_{k+1}} \backslash G_{M^{k+1}}(u)\right) \leq m_{0}^{k} \mu\left(\Omega_{\alpha_{1}} \backslash G_{M}(u)\right)+C_{n} k m_{0}^{k+1} \leq C m_{0}^{k+1}(1+k),
$$

where the constant $C$ depends on $\mu(\Omega)$. Writing $m_{1}=\sqrt{m_{0}}$ and since $m_{0}<1$, we have that $m_{0}^{k+1}(1+k) \leq \tilde{C} m_{1}^{k+1}$. So

$$
\mu\left(\Omega_{\alpha_{k+1}} \backslash G_{M^{k+1}}(u)\right) \leq \tilde{C} m_{1}^{k+1} .
$$

On the other hand, $\alpha_{k+1}=\alpha_{1}-\sum_{j=1}^{k}\left(\frac{\theta^{3}}{C_{0} M^{j}}\right)^{1 / \gamma} \geq \alpha_{1}-\sum_{j=1}^{\infty}\left(\frac{\theta^{3}}{C_{0} M^{j}}\right)^{1 / \gamma}=\alpha_{1}-$ $\left(\frac{\theta^{3}}{C_{0}}\right)^{1 / \gamma} \frac{1}{M^{1 / \gamma}-1} \geq \alpha_{0} / 2$, for $M$ sufficiently large depending on $\alpha_{1}$, and therefore

$$
\mu\left(\Omega_{\alpha_{0} / 2} \backslash G_{M^{k+1}}(u)\right) \leq \tilde{C} m_{1}^{k+1}
$$

for $k=1,2, \cdots$.

If $t \geq M$, pick $k$ such that $M^{k+1} \leq t<M^{k+2}$; then $k+1 \leq \log _{M} t<k+2$ and

$$
\mu\left(\Omega_{\alpha_{0} / 2} \backslash G_{t}(u)\right) \leq \mu\left(\Omega_{\alpha_{0} / 2} \backslash G_{M^{k+1}}(u)\right) \leq \tilde{C} m_{1}^{k+1} \leq \frac{C}{m_{1}} t^{\log _{M} m_{1}} .
$$


Combining this inequality with Remark 3.8 and (3.5) we get the estimate

$$
\mu\left(\left\{x \in \Omega_{\alpha_{0} / 2}:\left|D_{i j} u(x)\right|>\beta\right\}\right) \leq \frac{C}{\beta^{\tau}},
$$

for some $\tau>0$ and for all $\beta$ large. Therefore, we obtain the main theorem in the nonhomogeneous case.

Theorem 6.3. Let $\Omega$ be a normalized convex domain, $\phi \in C^{2}(\Omega)$ convex, $\phi=0$ on $\partial \Omega, \lambda \leq \operatorname{det} D^{2} \phi \leq \Lambda, u \in C^{2}(\Omega)$, and $L_{\phi} u=f$ in $\Omega$. Then, given $0<\alpha_{0}<1$ there exist positive constants $p$ and $C$ depending only on $\lambda, \Lambda$ and $\alpha_{0}$ such that

$$
\left\|D^{2} u\right\|_{L^{p}\left(\Omega_{\alpha_{0} / 2}\right)} \leq C\left(\|u\|_{L^{\infty}(\Omega)}+\|f\|_{L^{n}(\Omega)}\right) .
$$

Proof. Let $v=\frac{u}{\|u\|_{L^{\infty}(\Omega)}+\left\|\frac{f}{\operatorname{det} D^{2} \phi}\right\|_{L^{n}(\Omega, d \mu)}}$. Applying (6.7) to $v$, we obtain $\left\|D^{2} v\right\|_{L^{p}\left(\Omega_{\alpha_{0} / 2}, d \mu\right)} \leq C$ for some $p$ and $C$ depending on $\lambda, \Lambda$, and $\alpha_{0}$. This implies that

$$
\left\|D^{2} u\right\|_{L^{p}\left(\Omega_{\alpha_{0} / 2}, d \mu\right)} \leq C\left(\|u\|_{L^{\infty}(\Omega)}+\|f\|_{L^{n}(\Omega, d \mu)}\right) .
$$

The theorem then follows, since by assumption Lebesgue measure is comparable to the Monge-Ampère $\mu$.

Remark 6.4. Suppose $\lambda \leq M \phi=f \leq \Lambda$ in $\Omega$ with $\phi=0$ on $\partial \Omega$. If $f \in C^{\alpha}(\Omega)$, then from [C] Theorem 2] we have that $\phi \in C^{2, \alpha}(\Omega)$. Then $\lambda^{\prime} I d \leq D^{2} \phi(x) \leq \Lambda^{\prime} I d$ for all $x \in \Omega^{\prime} \Subset \Omega$ and with $\lambda^{\prime}$ and $\Lambda^{\prime}$ positive constants depending only on $n, \lambda, \Lambda$, dist $\left(\Omega^{\prime}, \partial \Omega\right)$ and the $C^{\alpha}$-norm of $f$. Suppose $L_{\phi} u=0$ in $\Omega$. Then by classical Schauder's estimates $u \in C^{2, \alpha}\left(\Omega^{\prime \prime}\right)$ for $\Omega^{\prime \prime} \Subset \Omega^{\prime}$, in particular, $\left\|D^{2} u\right\|_{L^{p}\left(\Omega^{\prime \prime}\right)} \leq C$ for all $p>0$ where the constant $C$ depends on $n, \lambda, \Lambda$, $\operatorname{dist}\left(\Omega^{\prime \prime}, \partial \Omega^{\prime}\right)$ and the $C^{\alpha}$-norm of $f$.

\section{Convexity inequality}

Let $u, f \in C(\Omega)$ and $f \geq 0$. We say that $M u \leq f$ in the viscosity sense if $u$ is a viscosity supersolution to $M u=f$ in $\Omega$.

Theorem 7.1. Let $u, v \in C(\bar{\Omega})$ convex, with $M u \leq 1+\epsilon$ in the viscosity sense, and $v$ a generalized solution to $M v=\alpha$ in $\Omega$, with $\alpha$ a positive constant. Let $\Gamma(u-v)$ be the convex envelope of $u-v$ in $\Omega$. Then

$$
|\partial \Gamma(u-v)(E)| \leq \int_{\mathcal{C} \cap E}\left(\left((1+\epsilon)^{1 / n}-\alpha^{1 / n}\right)^{+}\right)^{n} d x,
$$

for each Borel set $E \subset \Omega$, where $\mathcal{C}=\{x \in \Omega: \Gamma(u-v)(x)=(u-v)(x)\}$. If $v \in C^{2}(\Omega)$ is convex, then

$$
|\partial \Gamma(u-v)(E)| \leq \int_{\mathcal{C} \cap E}\left(\left((1+\epsilon)^{1 / n}-\operatorname{det} D^{2} v(x)^{1 / n}\right)^{+}\right)^{n} d x .
$$

We use the following lemma.

Lemma 7.2. If $u \in C(\bar{\Omega}), \Gamma(u)$ is the convex envelope of $u$ in $\Omega$, and $\mu$ is the Monge-Ampère measure associated with $\Gamma(u)$, then $\mu$ has support in the contact set $\mathcal{C}=\{x \in \Omega: \Gamma(u)(x)=u(x)\}$. 
Proof. Let $B \subset \Omega$ be a Borel set. We have $\mu(B)=\mu(B \cap \mathcal{C})+\mu(B \cap(\Omega \backslash \mathcal{C}))$. If $p \in \partial \Gamma(u)(\Omega \backslash \mathcal{C})$, then there exists $x_{0} \in \Omega \backslash \mathcal{C}$ with $p \in \partial \Gamma(u)\left(x_{0}\right)$, and so $\ell(x)=\Gamma(u)\left(x_{0}\right)+p \cdot\left(x-x_{0}\right)$ is a supporting hyperplane to $\Gamma(u)$ at $x_{0}$ in $\Omega$. By G01, Lemma 6.6.2] there exist at most $n+1$ points $x_{i} \in \mathcal{C}$ such that $x_{0}=\sum_{i=1}^{n+1} \lambda_{i} x_{i}$ with $\lambda_{i} \geq 0, \sum_{i=1}^{n+1} \lambda_{i}=1$, and $\Gamma(u)\left(x_{i}\right)=u\left(x_{i}\right)=\ell\left(x_{i}\right)$. So $\Gamma(u)(z)=\ell(z)$ for each $z$ in the simplex generated by $\left\{x_{i}\right\}_{i=1}^{n+1}$, and in particular for some $z \neq x_{0}$, that is, $p \in \partial \Gamma(u)(z)$. Then by Aleksandrov [G01, Lemma 1.1.12], $|\partial \Gamma(u)(\Omega \backslash \mathcal{C})|=0$.

Proof of Theorem [7.1. We proceed in a sequence of steps.

Step 1. Let $B=B_{R}(y)$ be a ball with $\bar{B} \subset \Omega$, and $u \in C(\Omega)$. There exists a sequence $g_{k} \in C^{\infty}(\partial B)$ such that $\left|g_{k}(x)-u(x)\right| \leq 1 / k$ for each $x \in \partial B$. Let $u_{k}$ be the convex solution to $\operatorname{det} D^{2} u_{k}=1+\epsilon$ in $B, u_{k}=g_{k}$ in $\partial B$, and $u_{k} \epsilon$ $C^{\infty}(B) \cap C(\bar{B})$. A variant of G01, Lemma 1.6.1] gives that there exist a subsequence of $u_{k}$, also denoted by $u_{k}$, and $u_{\infty} \in C(\bar{B})$ convex such that $u_{k} \rightarrow u_{\infty}$ uniformly on compact subsets of $B$, with $M u_{\infty}=1+\epsilon$ in the generalized sense in $B$ and $u_{\infty}=u$ on $\partial B$. Since $\operatorname{det} D^{2} u_{k}=M u$ in the generalized sense, by the comparison principle [G01, Theorem 1.4.6] we have that

$$
\min _{B}\left(u_{k}-u_{\infty}\right)=\min _{\partial B}\left(g_{k}-u\right) \text { and } \max _{B}\left(u_{k}-u_{\infty}\right)=\max _{\partial B}\left(g_{k}-u\right) .
$$

Therefore $\left|u_{k}-u_{\infty}\right| \leq 1 / k$ in $\bar{B}$, and so $u_{k}$ converges uniformly to $u_{\infty}$ in $\bar{B}$.

Step $\mathbf{1}^{\prime}$. Let $v$ be a convex generalized solution to $M v=\alpha$ in $\Omega$. There exists $h_{k} \in C^{\infty}(\partial B)$ such that $\left|h_{k}(x)-v(x)\right| \leq 1 / k$ in $\partial B$. Let $v_{k}$ be the solution to $\operatorname{det} D^{2} v_{k}=\alpha$ in $B$ with $v_{k}=h_{k}$ on $\partial B$. We have that $v_{k} \in C^{2}(B)$. Since $M v=\operatorname{det} D^{2} v_{k}=\alpha$ in $B$, by the comparison principle $\left|v_{k}(x)-v(x)\right| \leq 1 / k$ for all $x \in B$. Therefore $v_{k} \rightarrow v$ uniformly in $\bar{B}$.

Step 2. If $M u \leq 1+\epsilon$ in the viscosity sense in a ball $B=B_{R}(y)$ with $u \in C(\bar{B})$, then $u_{\infty} \leq u$ in $\bar{B}$. Let $\delta>0$ and $\phi(x)=\delta|x-y|^{2}$. Expanding the determinant we obtain

$$
\operatorname{det} D^{2}\left(u_{k}+\phi\right) \geq \operatorname{det} D^{2} u_{k}+(2 \delta)^{n}>1+\epsilon \geq M u .
$$

Then from [G01, Lemma 1.7.2] we get that

$$
\min _{B}\left(u-u_{k}-\phi\right)=\min _{\partial B}\left(u-g_{k}-\delta R^{2}\right) \geq-1 / k-\delta R^{2} .
$$

Hence $u(x) \geq u_{k}(x)+\delta|x-y|^{2}-\frac{1}{k}-\delta R^{2}$ for $x \in \bar{B}$. Letting $\delta \rightarrow 0$ and $k \rightarrow \infty$ we get $u \geq u_{\infty}$.

Step 3. We have $\Gamma_{B}\left(u_{k}-v_{k}\right) \rightarrow \Gamma_{B}\left(u_{\infty}-v\right)$ uniformly in $\bar{B}$; here $\Gamma_{B}$ denotes the convex envelope in $B$. This follows from Steps $1-1^{\prime}$.

Step 4. Since $u \geq u_{\infty}$ in $\bar{B}$ and $u=u_{\infty}$ on $\partial B$, we have that

$$
\left|\partial \Gamma_{\Omega}(u-v)(B)\right| \leq\left|\partial \Gamma_{B}\left(u_{\infty}-v\right)(B)\right|,
$$

where $\Gamma_{\Omega}$ and $\Gamma_{B}$ denote the convex envelopes in $\Omega$ and in $B$, respectively, for any $v \in C(\bar{\Omega})$. In fact, from Lemma 7.2

$$
\left|\partial \Gamma_{\Omega}(u-v)(B)\right|=\left|\partial \Gamma_{\Omega}(u-v)(B \cap \mathcal{C})\right|,
$$

where $\mathcal{C}=\left\{x \in \Omega: \Gamma_{\Omega}(u-v)(x)=(u-v)(x)\right\}$. Let $p \in \Gamma_{\Omega}(u-v)(B \cap \mathcal{C})$. Then $p \in \partial\left(u_{\infty}-v\right)(B)$. Indeed, there exists $y \in B \cap \mathcal{C}$ such that $\Gamma_{\Omega}(u-v)(x) \geq$ $\Gamma_{\Omega}(u-v)(y)+p \cdot(x-y)$ for all $x \in \Omega$. Since $\Gamma_{\Omega}(u-v)(x) \leq(u-v)(x)$ for all $x \in \Omega$, we obtain $(u-v)(x) \geq(u-v)(y)+p \cdot(x-y)$ for all $x \in \Omega$, i.e., $p \in \partial(u-v)(x)$. Since $u=u_{\infty}$ on $\partial \Omega$, we get from [G01, Lemma 1.4.1] that $p \in \partial\left(u_{\infty}-v\right)(B)$. We now need the following: if $h \in C(\bar{D})$ with $D$ strictly convex, 
then $\Gamma_{D}(h)(x)=h(x)$ for all $x \in \partial D$ (this follows arguing as in the first part of the proof of [G01, Theorem 1.5.2]; or noticing that $\Gamma_{D}(h)(x)=\Gamma_{\text {affine }}(h)(x)$ for $x \in D$, where $\Gamma_{\text {affine }}(h)(x)=\sup \{a(x): a$ is affine and $a(x) \leq h(x)$ for all $x \in \partial D\}$, and then applying the first part of [G01, Theorem 1.5.2] directly). Applying this fact with $h=u_{\infty}-v$ and $D=B$, we get that $\Gamma_{B}\left(u_{\infty}-v\right)=u_{\infty}-v$ in $\partial B$ and since $\Gamma_{B}\left(u_{\infty}-v\right) \leq u_{\infty}-v$ in $B$, it follows once again from [G01, Lemma 1.4.1] that $\partial\left(u_{\infty}-v\right)(B) \subset \partial \Gamma_{B}\left(u_{\infty}-v\right)(B)$, and Step 4 is complete.

Step 5. We claim that

$$
\left|\partial \Gamma_{B}\left(u_{\infty}-v\right)(B)\right| \leq \liminf _{k \rightarrow \infty}\left|\partial \Gamma_{B}\left(u_{k}-v_{k}\right)(B)\right| .
$$

From Step 3 and [G01, Lemma 1.2.2 (ii)], we have

$$
\left|\partial \Gamma_{B}\left(u_{\infty}-v\right)(K)\right| \leq \liminf _{k \rightarrow \infty}\left|\partial \Gamma_{B}\left(u_{k}-v_{k}\right)(U)\right|
$$

for each $K \subset U \subset \bar{U} \subset B$ with $K$ compact and $U$ open. Since the Monge-Ampère measure is a Borel measure finite on compact sets, it is regular, and so

$$
\left|\partial \Gamma_{B}\left(u_{\infty}-v\right)(B)\right|=\sup _{K \subset B, K \mathrm{cpt}}\left|\partial \Gamma_{B}\left(u_{\infty}-v\right)(K)\right|,
$$

and the claim follows.

Step 6. We have

$$
\left|\partial \Gamma_{B}\left(u_{k}-v_{k}\right)(B)\right| \leq \int_{\mathcal{C}_{k}} \operatorname{det} D^{2}\left(u_{k}-v_{k}\right)(x) d x
$$

where $\mathcal{C}_{k}=\left\{x \in B: \Gamma_{B}\left(u_{k}-v_{k}\right)(x)=\left(u_{k}-v_{k}\right)(x)\right\}$, from [G01, Theorem 1.4.5]. For $x \in \mathcal{C}_{k}$ we have

$$
\left(\operatorname{det} D^{2} v_{k}(x)\right)^{1 / n}+\left(\operatorname{det} D^{2}\left(u_{k}-v_{k}\right)(x)\right)^{1 / n} \leq\left(\operatorname{det} D^{2} u_{k}(x)\right)^{1 / n},
$$

and hence

$$
\begin{aligned}
\int_{\mathcal{C}_{k}} \operatorname{det} D^{2}\left(u_{k}-v_{k}\right)(x) d x & \leq \int_{\mathcal{C}_{k}}\left((1+\epsilon)^{1 / n}-\left(\operatorname{det} D^{2} v_{k}(x)\right)^{1 / n}\right)^{n} d x \\
& \leq \int_{B}\left(\left((1+\epsilon)^{1 / n}-\alpha^{1 / n}\right)^{+}\right)^{n} d x
\end{aligned}
$$

for each ball $B \subset \Omega$. Combining Steps $4-5$ and (7.3), we get that

$$
\left|\partial \Gamma_{\Omega}(u-v)(B)\right| \leq\left(\left((1+\epsilon)^{1 / n}-\alpha^{1 / n}\right)^{+}\right)^{n}|B|
$$

for each ball $B \subset \Omega$. Therefore, the measure $\left|\partial \Gamma_{\Omega}(u-v)(\cdot)\right|$ is absolutely continuous with respect to Lebesgue measure, and so there exists $h \in L_{l o c}^{1}(\Omega)$ such that

$$
\left|\partial \Gamma_{\Omega}(u-v)(E)\right|=\int_{E} h(x) d x
$$

for each Borel set $E \subset \Omega$, and

$$
h(x) \leq\left(\left((1+\epsilon)^{1 / n}-\alpha^{1 / n}\right)^{+}\right)^{n}, \quad \text { a.e. in } \Omega .
$$

Then (7.1) follows from Lemma 7.2

The proof of (7.2) follows omitting Step $1^{\prime}$ and taking $v_{k}=v$. 


\section{Counterexamples}

We consider a slight variant of the two-dimensional example from [W95, p. 842]. Let $\alpha \geq 4$ and

$$
\phi(x, y)= \begin{cases}x^{\alpha}+\beta y^{2} x^{2-\alpha}, & \text { if }|y| \leq|x|^{\alpha-1} \\ \gamma x^{2} y^{(\alpha-2) /(\alpha-1)}+\delta y^{\alpha /(\alpha-1)}, & \text { if }|y|>|x|^{\alpha-1}\end{cases}
$$

where $\beta, \gamma$ and $\delta$ are constants that will be chosen such that $\phi$ and $D \phi$ are continuous across the curve $|y|=|x|^{\alpha-1}, \phi$ is convex in the regions $|y|>|x|^{\alpha-1}$ and $|y|<$ $|x|^{\alpha-1}$, and $C_{1}(\alpha) \leq M \phi \leq C_{2}(\alpha)$ in the generalized sense, where $C_{i}(\alpha)$ are positive constants depending only on $\alpha$.

We have

$$
\begin{gathered}
\phi_{x}(x, y)= \begin{cases}\alpha x^{\alpha-1}+\beta(2-\alpha) y^{2} x^{1-\alpha}, & \text { if }|y|<|x|^{\alpha-1}, \\
2 \gamma x y^{(\alpha-2) /(\alpha-1)}, & \text { if }|y|>|x|^{\alpha-1},\end{cases} \\
\phi_{y}(x, y)= \begin{cases}2 \beta y x^{2-\alpha}, & \text { if }|y|<|x|^{\alpha-1}, \\
\gamma \frac{\alpha-2}{\alpha-1} x^{2} y^{-1 /(\alpha-1)}+\delta \frac{\alpha}{\alpha-1} y^{1 /(\alpha-1)}, & \text { if }|y|>|x|^{\alpha-1},\end{cases} \\
\phi_{x x}(x, y)= \begin{cases}\alpha(\alpha-1) x^{\alpha-2}+\beta(2-\alpha)(1-\alpha) y^{2} x^{-\alpha}, & \text { if }|y|<|x|^{\alpha-1}, \\
2 \gamma y^{(\alpha-2) /(\alpha-1)}, & \text { if }|y|>|x|^{\alpha-1},\end{cases} \\
\phi_{y y}(x, y) \\
\phi_{x y}(x, y)= \begin{cases}2 \beta(2-\alpha) y x^{1-\alpha}, & \text { if }|y|<|x|^{\alpha-1}, \\
2 \gamma \frac{\alpha-2}{\alpha-1} y^{-1 /(\alpha-1)}, & \text { if }|y|>|x|^{\alpha-1},\end{cases} \\
= \begin{cases}2 \beta x^{2-\alpha}, & \text { if }|y|<|x|^{\alpha-1}, \\
-\gamma \frac{\alpha-2}{\alpha-1} \frac{1}{\alpha-1} x^{2} y^{-\alpha /(\alpha-1)}+\delta \frac{\alpha}{\alpha-1} \frac{1}{\alpha-1} y^{(2-\alpha) /(\alpha-1)}, & \text { if }|y|>|x|^{\alpha-1} .\end{cases}
\end{gathered}
$$

Therefore

$$
2 \beta\left(\alpha(\alpha-1) \geq \operatorname{det} D^{2} \phi \geq 2 \beta(\alpha(\alpha-1)-\beta(\alpha-2)(\alpha-3))\right.
$$

for $|y|<|x|^{\alpha-1}$, and

$$
\frac{2 \gamma \alpha \delta}{(\alpha-1)^{2}} \geq \operatorname{det} D^{2} \phi \geq \frac{2 \gamma}{(\alpha-1)^{2}}(\delta \alpha-\gamma(\alpha-2)(2 \alpha-3))
$$

for $|y|>|x|^{\alpha-1}$.

We now study the continuity of $\phi$ and $D \phi$ across $|y|=|x|^{\alpha-1}$. For the continuity of $\phi$ we need

$$
x^{\alpha}+\beta x^{2 \alpha-2} x^{2-\alpha}=\gamma x^{2} x^{\alpha-2}+\delta x^{\alpha},
$$

SO

$$
1+\beta=\gamma+\delta
$$

For the continuity of $\phi_{x}$ we need

$$
\alpha x^{\alpha-1}+\beta(2-\alpha) x^{2 \alpha-2} x^{1-\alpha}=2 \gamma x x^{\alpha-2},
$$

SO

$$
\alpha+\beta(2-\alpha)=2 \gamma
$$


For the continuity of $\phi_{y}$ we need

so

$$
2 \beta x^{\alpha-1} x^{\alpha-2}=\gamma \frac{\alpha-2}{\alpha-1} x^{2} x^{-1}+\frac{\delta \alpha}{\alpha-1} x,
$$

$$
2 \beta=\gamma \frac{\alpha-2}{\alpha-1}+\frac{\delta \alpha}{\alpha-1} .
$$

Therefore $\beta, \delta, \gamma$ solve the system of equations

$$
\begin{aligned}
\beta-\delta-\gamma & =1, \\
(2-\alpha) \beta-2 \gamma & =-\alpha, \\
2(\alpha-1) \beta-\alpha \delta-(\alpha-2) \gamma & =0 .
\end{aligned}
$$

The solutions to this system are given by

$$
(\beta, \delta, \gamma)=\left(1-\frac{2}{\alpha}, 0,2-\frac{2}{\alpha}\right)+\delta\left(\frac{2}{\alpha}, 1, \frac{2}{\alpha}-1\right),
$$

where $\delta$ is arbitrary.

We next impose conditions on $\beta, \delta, \gamma$ such that $\phi$ is convex. We have for $|y|<$ $|x|^{\alpha-1}$ that

$$
\phi_{x x}=(\alpha-1)\left(\alpha x^{\alpha-2}+\beta(\alpha-2) y^{2} x^{-\alpha}\right)>0
$$

if $\beta>0$. If $|y|>|x|^{\alpha-1}$, then

$$
\phi_{x x}=2 \gamma y^{(\alpha-2) /(\alpha-1)}>0
$$

for $\gamma>0$. Also, we have for $|y|<|x|^{\alpha-1}$ that

$$
\phi_{y y}=2 \beta x^{2-\alpha}>0
$$

for $\beta>0$. If $|y|>|x|^{\alpha-1}$, then

$$
\begin{aligned}
\phi_{y y} & =\frac{1}{(\alpha-1)^{2}}\left(\delta \alpha y^{(2-\alpha) /(\alpha-1)}-\gamma(\alpha-2) x^{2} y^{-\alpha /(\alpha-1)}\right) \\
& \geq \frac{1}{(\alpha-1)^{2}} y^{(2-\alpha) /(\alpha-1)}(\delta \alpha-\gamma(\alpha-2)) .
\end{aligned}
$$

Since $\gamma=2-\frac{2}{\alpha}+\delta\left(\frac{2}{\alpha}-1\right)$, we get that $\delta \alpha-\gamma(\alpha-2)>0$, and so $\phi_{x x}>0$, as long as

$$
\delta>\frac{2(\alpha-2)(\alpha-1)}{\alpha^{2}+(\alpha-2)^{2}} .
$$

We need $\operatorname{det} D^{2} \phi>0$. The right-hand side of (8.1) is positive if $\beta<\frac{\alpha(\alpha-1)}{(\alpha-2)(\alpha-3)}$, and since $\beta=1-\frac{2}{\alpha}+\delta \frac{2}{\alpha}$, we must have

$$
\delta<\frac{\alpha^{2}(\alpha-1)-(\alpha-2)^{2}(\alpha-3)}{2(\alpha-2)(\alpha-3)} .
$$

On the other hand, the right-hand side of (8.2) is positive if $\delta \alpha-\gamma(\alpha-2)(2 \alpha-3)>0$, and since $\gamma=2-\frac{2}{\alpha}+\delta\left(\frac{2}{\alpha}-1\right)$, we get

$$
\delta>\frac{2(\alpha-2)(\alpha-1)(2 \alpha-3)}{\alpha^{2}+(\alpha-2)^{2}(2 \alpha-3)} .
$$

Also $\gamma>0$ amounts to

$$
\delta<\frac{2(\alpha-1)}{\alpha-2}
$$


We have

$$
\begin{aligned}
\delta_{2}(\alpha)= & \min \left\{\frac{2(\alpha-1)}{\alpha-2}, \frac{\alpha^{2}(\alpha-1)-(\alpha-2)^{2}(\alpha-3)}{2(\alpha-2)(\alpha-3)}\right\}=\frac{2(\alpha-1)}{\alpha-2}, \\
\delta_{1}(\alpha)= & \max \left\{\frac{2(\alpha-2)(\alpha-1)}{\alpha^{2}+(\alpha-2)^{2}}, \frac{2(\alpha-2)(\alpha-1)(2 \alpha-3)}{\alpha^{2}+(\alpha-2)^{2}(2 \alpha-3)}\right\} \\
= & \frac{2(\alpha-2)(\alpha-1)(2 \alpha-3)}{\alpha^{2}+(\alpha-2)^{2}(2 \alpha-3)},
\end{aligned}
$$

and it is easy to check that $\delta_{1}(\alpha)<\delta_{2}(\alpha)$. Therefore each $\delta$ satisfying

$$
\delta_{1}(\alpha)<\delta<\delta_{2}(\alpha)
$$

determines $\beta$ and $\gamma$ with the desired properties and therefore $\phi$.

We have that $D^{2} \phi \notin L^{p}$ for $p>\frac{\alpha}{\alpha-2}$.

Lemma 8.1. Given $p>1$ there exist positive constants $\lambda \leq \Lambda$ depending only on $p$ and a strictly convex bounded domain $\Omega$ and a convex function $\psi \in C^{2}(\Omega)$ with $\lambda \leq \operatorname{det} D^{2} \psi \leq \Lambda$ in $\Omega$ and with $\psi=1$ on $\partial \Omega$ such that the inequality $\left\|D^{2} \psi\right\|_{L^{p}} \leq C$ is impossible with a constant $C$ depending only on $n, p, \lambda$ and $\Lambda$.

Proof. Let $\phi$ be the function constructed above for $\alpha \geq 4$ sufficiently large such that $p>\frac{\alpha}{\alpha-2}, \Omega=\{z: \phi(z)<1\}, f(z)=\operatorname{det} D^{2} \phi(z)$, and $\lambda=C_{1}(\alpha), \Lambda=C_{2}(\alpha)$. Consider $\eta_{\epsilon}(z)$ a nonnegative smooth mollifier and let $f_{\epsilon}=f \star \eta_{\epsilon}$. We have that $\lambda \leq f_{\epsilon} \leq \Lambda$ in $\Omega$. Let $\phi_{\epsilon}$ be the solution to $\operatorname{det} D^{2} \phi_{\epsilon}=f_{\epsilon}$ in $\Omega$ and $\phi_{\epsilon}=1$ on $\partial \Omega$. We have that $f_{\epsilon} \rightarrow f$ weakly. Then by [G01, Lemma 1.6.1], $\left\{\phi_{\epsilon}\right\}$ contains a subsequence $\phi_{\epsilon^{\prime}} \rightarrow \phi$. If $\left\|D^{2} \phi_{\epsilon^{\prime}}\right\|_{L^{p}} \leq C$ with a constant depending only on $p, n, \lambda$ and $\Lambda$, then $D^{2} \phi_{\epsilon^{\prime}}$ would contain a subsequence $D^{2} \phi_{\epsilon^{\prime \prime}}$ converging in $L^{p}$ to some function $v$. But then $D^{2} \phi=v$, a contradiction.

\section{REFERENCES}

[C] Caffarelli, L. A. 1990. Interior $W^{2, p}$ estimates for solutions to the Monge-Ampère equation. Ann. of Math. 131, 135-150. MR1038360 (91f:35059)

[C90] Caffarelli, L. A. 1990. A localization property of viscosity solutions to the Monge-Ampère equation and their strict convexity. Ann. of Math. 131, 129-134. MR 1038359(91f:35058)

[C91] Caffarelli, L. A. 1991. Some regularity properties of solutions of Monge-Ampère equation. Comm. Pure Appl. Math. 44, 965-969. MR1127042 (92h:35088)

[CC95] Cafarelli, L. A. and Cabré, X. 1995. Fully nonlinear elliptic equations American Mathematical Society Colloquium Publications, volume 43. MR.1351007 (96h:35046)

[CG96] Caffarelli, L. A. and Gutiérrez, C. E. 1996. Real analysis related to the Monge-Ampère equation. Trans. A. M. S. 348(3), 1075-1092. MR1321570 (96h:35047)

[CG97] Caffarelli L. A. and Gutiérrez C. E. 1997. Properties of the solutions of the linearized Monge-Ampère equation. Amer. J. Math. 119(2), 423-465. MR1439555 (98e:35060)

[E85] Evans, L. C. 1985. Some estimates for nondivergence structure, second order elliptic equations. Trans. A. M. S. 287(2), 701-712. MR0768735 (86g:35056)

[GT83] D. Gilbarg and N. S. Trudinger 1983. Elliptic Partial Differential Equations of Second Order. Springer-Verlag, New York. MR0737190 (86c:35035)

[G01] C. E. Gutiérrez 2001. The Monge-Ampère Equation. Birkhaüser, Boston. MR1829162 (2002e:35075)

[GH00] C. E. Gutiérrez and Qingbo Huang 2000. Geometric properties of the sections of solutions to the Monge-Ampère equation. Trans. A. M. S. 352, 4381-4396. MR1665332 (2000m:35060)

[L86] Fang-Hua Lin 1986. Second derivative $L^{p}$-estimates for elliptic equations of nondivergent type. Proc. Amer. Math. Soc. 96(3), 447-451. MR0822437 (88b:35058) 
[P78] Pogorelov, A.V. 1978. The Minkowski Multidimensional Problem. V.H. Winston and Sons, Washington. MR0478079 (57:17572)

[Sc93] Schneider, R. 1993. Convex Bodies: The Brunn-Minkowski Theory. Cambridge University Press, Cambridge. MR.1216521 (94d:52007)

[TW00] Trudinger, N. S. and Xu-Jia Wang 2000. The Bernstein problem for affine maximal hypersurfaces. Invent. Math. 140(2), 399-422. MR1757001 (2001h:53016)

[W95] Xu-Jia Wang 1995. Some counterexamples to the regularity of Monge-Ampère equations. Proc. Amer. Math. Soc. 123(3), 841-845. MR1223269 (95d:35025)

Department of Mathematics, Temple University, Philadelphia, Pennsylvania 19122

E-mail address: gutierrez@math.temple.edu

Department of Mathematics, Purdue University, West Lafayette, Indiana 47907-2067

E-mail address: fedeleti@aol.com

Current address: Instituto Argentino de Matemática, Saavedra 15, 1038 Buenos Aires, Argentina 